\title{
Ethnomedicinal knowledge of a marginal hill community of Central Himalaya: diversity, usage pattern, and conservation concerns
}

\author{
S. N. Ojha', Deepti Tiwari ${ }^{1}$, Aryan Anand ${ }^{1}$ and R. C. Sundriyal ${ }^{1,2^{*}}$
}

\begin{abstract}
Background: Indigenous communities use wild plants to cure human ailments since ancient times; such knowledge has significant potential for formulating new drugs and administering future health care. Considering this, the present study was undertaken to assess use value, diversity, and conservation concerns of medicinal plants used in traditional herbal care system of a marginal hill community in Bageshwar district of Uttarakhand in the Central Himalayan region of India.

Methodology: Extensive surveys were made in 73 villages to gather information on the ethnomedicinal use of plant species used in the traditional herbal healing system. A total of 100 respondents were identified (30 herbal healers called Vaidyas and 70 non-healers/natives) and interviewed using semi-structured questionnaires, target interviews, and group discussion. Some important indices such as the use-value index (UV), relative frequency citation (RFC), cultural importance index $(\mathrm{Cl})$, and informant consensus factor $\left(F_{i \mathrm{i}}\right)$ were calculated for the medicinal plants included in the present study.

Result: It was recorded that the community uses a total of 70 species with 64 genera and 35 families for curing various ailments. Family Lamiaceae recorded the maximum number of medicinal plants. Twenty-one species used most extensively in the traditional health care system. The major parts of the identified plants used for the treatment of various ailments were root/rhizome and leaf. The most common methods used for the preparation of these plants were decoction and infusion. Ocimum basilicum L., Cannabis sativa L., Citrus aurantifolia (Christm) Sw., Curcuma longa L., and Setaria italica L. had the highest rate of use report. RFC value ranged between 0.03 and 0.91 with highest values for Setaria italica, Zingiber officinale, Ocimum basilicum, and Raphanus sativus. The traditional knowledge is passed verbally to generations and needs to be preserved for the future bio-prospecting of plants that could be a potential cure to any future disease.

Conclusion: In recent years, the community has access to modern hospitals and medicinal facilities, although a considerable number still prefer medicinal plants for curing select ailments. It is suggested that these ethnomedicinal species need to be screened and evaluated further for their effectiveness for pharmacological activity. Also, significant efforts are required to conserve traditional knowledge and natural habitats of wild medicinal plants.
\end{abstract}

Keywords: Ethnomedicinal plants, Traditional knowledge, Indigenous people, Ailments, Public health, Bageshwar, Uttarakhand

\footnotetext{
* Correspondence: sundriyalrc@yahoo.com

${ }^{1}$ G.B. Pant National Institute of Himalayan Environment, Kosi-Katarmal,

Almora, Uttarakhand 263 643, India

${ }^{2}$ Department of Forestry and Natural Resources, HNB Garhwal University,

(Garhwal), Srinagar, Uttarakhand 246174, India
}

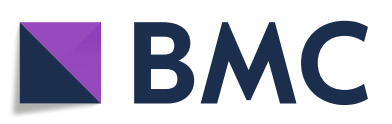

(c) The Author(s). 2020 Open Access This article is licensed under a Creative Commons Attribution 4.0 International License, which permits use, sharing, adaptation, distribution and reproduction in any medium or format, as long as you give appropriate credit to the original author(s) and the source, provide a link to the Creative Commons licence, and indicate if changes were made. The images or other third party material in this article are included in the article's Creative Commons licence, unless indicated otherwise in a credit line to the material. If material is not included in the article's Creative Commons licence and your intended use is not permitted by statutory regulation or exceeds the permitted use, you will need to obtain permission directly from the copyright holder. To view a copy of this licence, visit http://creativecommons.org/licenses/by/4.0/. The Creative Commons Public Domain Dedication waiver (http://creativecommons.org/publicdomain/zero/1.0/) applies to the data made available in this article, unless otherwise stated in a credit line to the data. 


\section{Background}

Medicinal plants have been utilized for the treatment of various diseases since ancient times, thus form an important element of aboriginal curative systems. The Indian Rishis first documented the use of medicinal plants in the form of Samhitas. Charak Samhita (1000-800 BC) and Shushrut Samhita (800-700 BC) by Maharshi Charak and and Maharshi Shashurut, respectively, are the baselines of the Indian Medicinal System. Maharshi Charak mentioned over 500 medicinal plants, out of which 340 plants used in the production of herbal medicine $[1,2]$. AYUSH (i.e., Ayurveda, Unani, Siddha, and Homeopathy) is another traditional Indian health care system that is considered a great knowledge base in herbal medicines. Ayurveda reports over 2000 medicinal plant species, Siddha 1121 plant species, Unani 751 species, and homeopathy 422 species [3]. Nearly $70-80 \%$ population worldwide still relies on traditional medicinal systems for their primary health care because of their effectiveness, cultural preferences, and lack of modern health care alternatives [4, 5]. The global demand for herbal medicine continues to increase over the past few decades. The earlier studies stated that out of 250,000 flowering plants in the world, only less than $10 \%$ have been screened so far for their medicinal potency, and still, 90\% remains unexplored [2]. In recent times, there is an increased interest regarding the use of the medicinal plants to develop new drugs and medicines for fulfilling the demand of a growing population [6-8]. Therefore, the information on plants of ethnomedicinal importance holds high potential. Uttarakhand Himalaya is a mountainous region in northern India that has a unique geography, rich biological resources, cultural heritage, and diverse climatic conditions which supports the highest number of medicinal plant species [9]. Over two-third population live in rural areas and depend on diverse natural resources to fulfill their need for food, fuel, fodder, timber, medicine, etc. Communities use a large variety of medicinal plants for treating diverse ailments $[10,11]$. However, it is strongly being realized that the indigenous knowledge related to herbal medicines is continuously being eroded despite high significance to humanity. The subject needs further research such as documentation of potential medicinal species, analyzing their active constituents, clinical trials for validations, and developing new drugs and medicines [8-12]. Considering this, the present study was undertaken. We argue that sustainable management and conservation of medicinal plants can be achieved when information about their use for treating ailments and traditional herbal practices within particular areas are available. Such information is strongly desired to be preserved from being lost for the use of both the present and the future generations. For the purpose of this study, we selected marginal community and local herbal practitioners (Vaidyas) of Bageshwar district in Uttarakhand state in north India and documented ethnomedicinal plant diversity and traditional medicinal practices being used by them. Efforts were also made to scientifically validate and interpret the data using several indices such as relative frequency citation (RFC), use report (categorical and disease-based), cultural importance index (CI), and informant consensus index (Fic) so as to verify the homogeneity, importance, and the cultural similarity of the medicinal plants in communities. It is expected that the qualitative and quantitative information generated from the study will have immense utility for the conservation and sustainable utilization of medicinal plants as well as for managing the traditional health care system.

\section{Materials and methods}

\section{Study area}

This study aimed to investigate the medicinal species used by the marginal hill community living in remote and high-altitude areas where medical health care facilities are not easily available. These practices are being used since eternity descended from the inherited knowledge of the locals and indigenous population of Uttarakhand. The study was carried out at Bageshwar district (geographical area $1687.8 \mathrm{~km}^{2}$ ) of Uttarakhand state and lies between latitudes $29^{\circ} 42^{\prime} 40^{\prime \prime}$ to $30^{\circ} 18^{\prime} 56^{\prime \prime} \mathrm{N}$ and longitudes $79^{\circ} 23^{\prime}$ to $80^{\circ} 10^{\prime} \mathrm{E}$ (Fig. 1). The district is situated on the confluence of Gomti river and Saryu river which is a tributary of Kali river. It is bounded by Almora district in the southwest, Chamoli district in the north and northwest, and Pithoragarh district in the east. Administratively, the district is divisible into four Tehsils, viz., Bageshwar, Kapkot, Kanda (Sub-tehsil), and Garur, and three blocks, viz., Bageshwar, Garur, and Kapkot. There are 947 revenue villages, out of which 874 villages are inhabited, and 73 villages are uninhabited. As per the 2011 census, the total population of Bageshwar district is 259,898 (male $48 \%$, female $52 \%$ ) with $96 \%$ living in the rural areas.

The community of the area is divided into 3 categories, viz., General, Scheduled Class (SC), Scheduled Tribe (ST), and majority of them involved in primary sector (agricultural activities), while some also work in secondary and tertiary sectors, such as private works, businesses, and government jobs. As such, the community is highly marginal with small and scattered land holdings, low production, and low income, therefore, highly dependent on natural resources. Male population outmigrates to earn better livelihoods that lead to continuous increase in fallow lands and culturable waste lands.

\section{Data collection}

The study was conducted in 39 villages covering Garur-Ganga valley (23 villages) and Saryu valley (16 


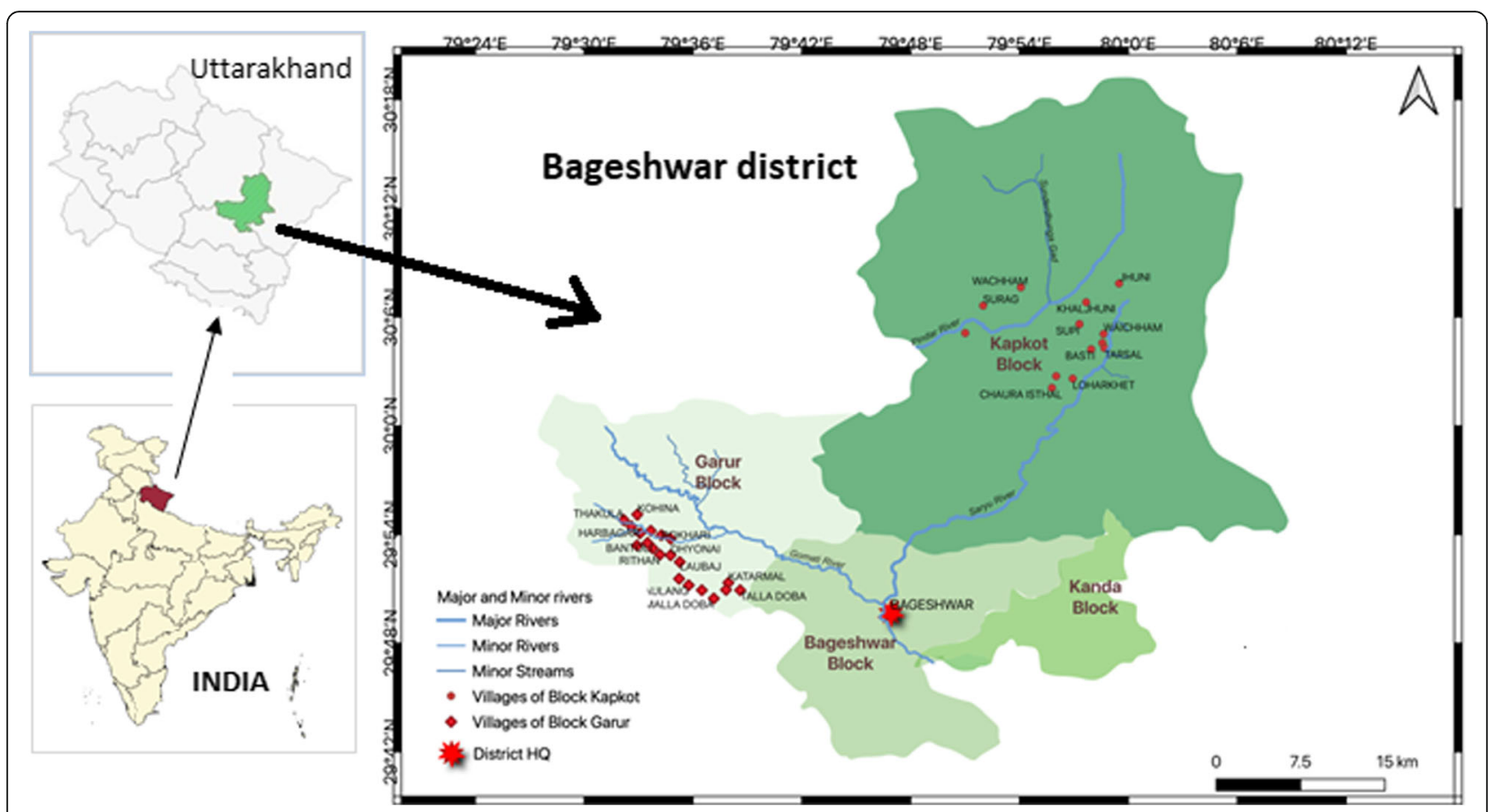

Fig. 1 Study area and villages in Garur and Kapkot Bolcks of District Bageshwar, Uttarakhand, India

villages) of Garur and Kapkot Blocks during 20162018. To fulfill the objectives of the study, extensive field visits were made to gather information from traditional herbal healers (Vaidyas) and indigenous people using semi-structured questionnaires, target interviews, and visual interpretation through snowball methodology. A total of 100 respondents were randomly selected for the present study from both valleys, 37 being male and 63 female respondents. Of them, 30 were Vaidyas (male 19, female 11). Female informants were given preference in view of their dominance in villages. The age group of informants varied between 30 and 83 years, although most of them were between 50 and 65 years of age (Fig. 2). The questionnaire contains information about the ethnomedicinal plants with their local name, parts used, habit, ailment treated by medicinal plants, and mode of utilization of herbal formulation. Two general meetings and interviews were also organized at each valley with Vaidyas and natives. The documented medicinal plant species were validated for identification using available literature [13-16]. The specimens matched with the herbarium lodged in CCRAS-RARI, Tarikhet, Ranikhet, Uttarakhand (acronym RKT), which houses largest medicinal plant herbariums in northern India. A few generally available species were matched with the plant database of Centre for Socio-Economic Development deposited at G.B. Pant National Institute of Himalayan Environment (GBP-NIHE), Almora, Uttarakhand.

\section{The ethnobotanical analysis}

The information on ethnomedicinal important species were recorded including the local names of the species, habit, their uses in different forms, the part used in the medical practice, mode of administration, and the condition of the plant (fresh or dry). The plants were classified into 12 main categories of ailments which were further divided into different respective subcategories based on disease and affected body part. The data were then statistically analyzed for different parameters. To enhance the indicative value of the ethnomedicinal study, suitable quantitative methods and approaches were used in the form of indices, such as relative frequency of citation (RFC), use report (based on illness, based on taxa), cultural importance (CI), and consensus factor of informants (Fic).

Use-report values (UR) provides information on the total number of reported uses for each species. It is similar to the use value of a species, but for use report, the number of events (interviews), the process of asking one informant on one day about the uses they know for one species, is one because the respondents were interviewed only once. And response use values are broken down by the number of uses reported for each plant species part.

Use-value index (UV) depicts the importance of each species for each informant and calculated by $U V=\Sigma U / N$ formula where $U$ is the number of uses quoted in each interview by $N$, number of informants. Use values are high when there are many useful reports for a plant representing its importance and come within reach to zero (0) when the use reports are low [17]. 


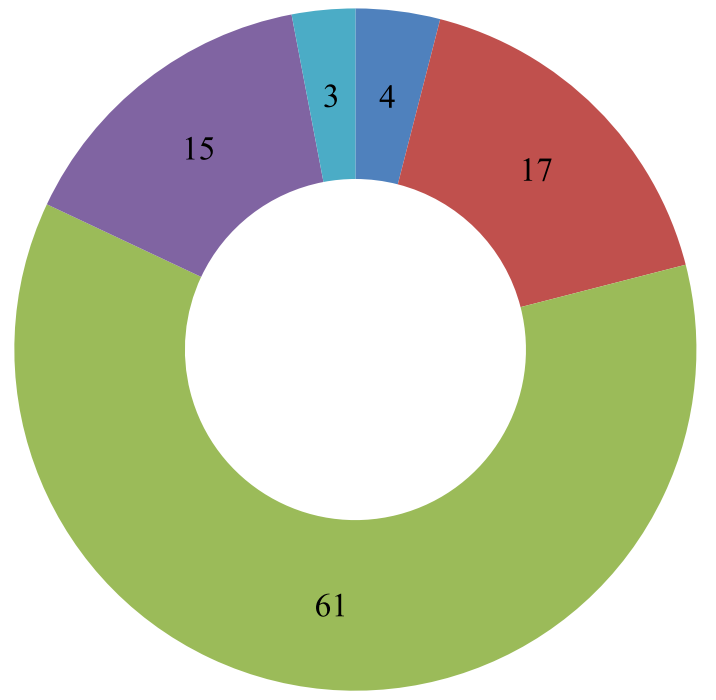

Below 35 years

35-50 years

51-65 years

66-80 years

Above 80 years

Fig. 2 Age distribution of respondents

Relative frequency citation (RFC) index reveals the usage importance of a particular species used by different informants. The index is calculated by dividing the total number of informants referring to a particular taxon with the total number of informants $(R F C=F C / N)$ where $F C$ is the total number of informants that referred to the taxon, and $N$ is the total number of informants [18].

Cultural importance index (CI) is estimated for each locality as the summation of Use-Report (UR) in every use category mentioned for a species in the locality divided by the total number of informants. This index provides an implication of the involvement of a particular taxon in the community, and a greater value signifies that a particular is widely distributed among communities. A null value indicates non-existence of the species in the area. CI is calculated as $C I=U R / N$ where $U R$ is the total number of use reports for each species in every category of illness mentioned, and $N$ is the total number of informants [19].

Informant consensus factor (Fic) is used to test the consistency of information knowledge in treating a particular illness category. The values obtained are near one (1) for welldefined selection criteria in the community and/or if the information is exchanged between the informants. A value approaching zero $(0)$ represents that the plants are chosen randomly, and/or there is no information exchanged between the communities about their use. Fic is calculated as Fic $=(N u r$ - Nt)/(Nur - 1), where Nur refers to the number of use reports for a particular use category, and $N t$ refers to the number of taxa used for a particular use category by all informants [20].

\section{Result and discussion}

\section{Ethnomedicinal uses of plants and mode of practice}

The residents of different age groups were surveyed to assess the ethnomedicinal uses of plant species (Fig. 2).
The survey revealed that a total of 70 medicinal plant species varying from 35 families and 64 genera have been used by the inhabitants of 39 villages for different (Table 1). Family Lamiaceae recorded maximum species (8) followed by Asteraceae (6 species), Fabaceae (5 species), Rosaceae (4 species), and Apiaceae, Liliaceae, Ranunculaceae, Rutaceae, and Zingiberaceae (3 species each). The remaining families were represented with just one or two species. Almost all the species are widely used by the community. Of the total documented medicinal plant species, the herbaceous habit (51 species) was the most dominant life form, followed by the tree (10), shrub (7), and climbers (2 species) (Fig. 3).

It was interesting to note that nearly $70 \%$ population still use prescription of Vaidyas for common ailments, although the Vaidyas were having an age of $>50$ years. The diseases cured by Vaidyas comprised fever, stomach problems, cough, cold, headache, etc. The most common plant parts used were root/rhizome, followed by leaf, whole plant, seeds, fruits, flower, and bulb and latex (Fig. 4). The collection of plant parts was very selective keeping into consideration the time of collection, plant matureness, and quantity of use thus ensuring a conservation approach. Vaidyas comprised sound knowledge and a species-specific method of preparing drugs to cure various ailments (Table 2). Making decoction and ingestion was the most common mode of plant part use (Fig. 5). Poultice and cooking were also favored for many medicinal plants. Another mode of application includes cooking and making into powder (9.42\%), direct application $(7.97 \%)$, emulsion and infusion $(5.80 \%)$, and ointment $(2.17 \%)$ (Fig. 5). A decoction is the most commonly used method to cure ailments in traditional herbal systems [21-25]. It is considered to extract all 
Table 1 Quantitative enumeration of ethnomedicinal plants used by marginal hill community of District Bageshwar

\begin{tabular}{|c|c|c|c|c|c|c|c|c|c|c|c|c|}
\hline Botanical name & $\begin{array}{l}\text { Local } \\
\text { name }\end{array}$ & $\begin{array}{l}\text { Voucher/ } \\
\text { ident. no. }\end{array}$ & Habit & $\begin{array}{l}\text { Part } \\
\text { used }\end{array}$ & $\begin{array}{l}\text { Popular ailment uses (group and } \\
\text { categories) }\end{array}$ & $\begin{array}{l}\text { Used } \\
\text { in }\end{array}$ & Preparation & $F C^{a}$ & $\mathrm{RFC}^{\mathbf{b}}$ & $U R^{c}$ & $U R^{d}$ & $\mathrm{Cl}^{\mathbf{e}}$ \\
\hline \multicolumn{13}{|l|}{ Family: Alliaceae } \\
\hline Allium sativum $\mathrm{L}$. & Lasan & GBPCSED1 & $\mathrm{H}$ & B & $\begin{array}{l}\text { Skeleton and muscles-joint pain } \\
\text { (arthritis) }\end{array}$ & $\mathrm{Hu}$ & $\mathrm{O}$ & 59 & 0.59 & 59 & 59 & 0.59 \\
\hline \multicolumn{13}{|l|}{ Family: Apiaceae } \\
\hline \multirow[t]{2}{*}{$\begin{array}{l}\text { Angelica glauca } \\
\text { Edgew. }\end{array}$} & Gandaraini & RKT 27789 & $\mathrm{H}$ & Rt & $\begin{array}{l}\text { Gastrointestinal—stomach ache, } \\
\text { vomiting }\end{array}$ & $\mathrm{Hu}$ & Po & 44 & 0.44 & 35 & 89 & 0.89 \\
\hline & & & & & $\begin{array}{l}\text { Other-spices and condiment, herbal } \\
\text { tea }\end{array}$ & & $C o, \operatorname{lnf}$ & & & 54 & & \\
\hline $\begin{array}{l}\text { Centella asiatica } \\
\text { L. }\end{array}$ & Brahmi & RKT 28186 & $\mathrm{H}$ & L & General health care - Headache & $\mathrm{Hu}$ & Po & 28 & 0.28 & 28 & 28 & 0.28 \\
\hline $\begin{array}{l}\text { Coriandrum } \\
\text { sativum L. }\end{array}$ & Dhaniya & RKT 28118 & $\mathrm{H}$ & $\mathrm{Sd}$ & Antidote-against poison & C & $\mathrm{Em}$ & 36 & 0.36 & 36 & 36 & 0.36 \\
\hline \multicolumn{13}{|l|}{ Family: Araceae } \\
\hline \multirow[t]{2}{*}{$\begin{array}{l}\text { Acorus calamus } \\
\mathrm{L} \text {. }\end{array}$} & Bojh/Buch & RKT 27965 & $\mathrm{H}$ & $\mathrm{Rh}$ & $\begin{array}{l}\text { Skeleton and muscles_-sprain, } \\
\text { inflammation }\end{array}$ & $\mathrm{Hu}$ & Pw, O & 55 & 0.55 & 21 & 74 & 0.74 \\
\hline & & & & & Other-insect repellent & । & $\mathrm{Da}$ & & & 53 & & \\
\hline \multicolumn{13}{|l|}{ Family: Asteraceae } \\
\hline $\begin{array}{l}\text { Ageratina } \\
\text { adenophora } \\
\text { (Spreng.) King \& } \\
\text { H. Rob. }\end{array}$ & $\begin{array}{l}\text { Nargadiya/ } \\
\text { Pagaljhad }\end{array}$ & RKT 22106 & $\mathrm{H}$ & L & Dermatological—cuts and wounds & $\mathrm{Hu}$ & Po & 80 & 0.8 & 80 & 80 & 0.8 \\
\hline $\begin{array}{l}\text { Artemisia } \\
\text { martima L. }\end{array}$ & Pati/Titpati & RKT 23793 & $\mathrm{H}$ & $\mathrm{L}$ & $\begin{array}{l}\text { Dermatological—cuts and wounds, } \\
\text { skin ailments }\end{array}$ & $\mathrm{Hu}$ & Po & 55 & 0.55 & 77 & 77 & 0.77 \\
\hline \multirow{3}{*}{$\begin{array}{l}\text { Saussurea costus } \\
\text { (Falc.) Lipsch. }\end{array}$} & Kut/Kuth & RKT 28203 & $\mathrm{H}$ & Rt & General health care-fever & $\mathrm{Hu}$ & Pw & 28 & 0.28 & 27 & 64 & 0.64 \\
\hline & & & & & Respiratory—cough & & Pw & & & 8 & & \\
\hline & & & & & $\begin{array}{l}\text { Gastrointestinal—stomach ache, } \\
\text { dysentery }\end{array}$ & & De & & & 29 & & \\
\hline \multirow{2}{*}{$\begin{array}{l}\text { Taraxacum } \\
\text { officinale Weber. }\end{array}$} & Dudhil & RKT 27817 & $\mathrm{H}$ & $L, R t$ & Antidote_-snake bite & $\mathrm{Hu}$ & $\ln , P_{0}$ & 50 & 0.5 & 13 & 39 & 0.39 \\
\hline & & & & & $\begin{array}{l}\text { Other-to increase lactation in } \\
\text { mulching animals }\end{array}$ & C & $\operatorname{lnf}$ & & & 26 & & \\
\hline \multirow[t]{2}{*}{ Tegetus erecta $\mathrm{L}$. } & Hazari & GBPCSED2 & $\mathrm{H}$ & L & $\begin{array}{l}\text { General health care-fever, ear } \\
\text { infection }\end{array}$ & $\mathrm{Hu}$ & Po & 51 & 0.51 & 46 & 61 & 0.61 \\
\hline & & & & & Dermatological—wounds & & Po & & & 15 & & \\
\hline \multicolumn{13}{|l|}{$\begin{array}{l}\text { Family: } \\
\text { Berberidaceae }\end{array}$} \\
\hline \multirow{2}{*}{$\begin{array}{l}\text { Berberis asiatica } \\
\text { Roxb. ex DC }\end{array}$} & Kilmori & RKT 22109 & $S$ & Rt & General health care-fever & $\mathrm{Hu}$ & Pw & 42 & 0.42 & 13 & 54 & 0.54 \\
\hline & & & & & Circulatory—diabetes & & Pw & & & 41 & & \\
\hline \multicolumn{13}{|c|}{ Family: Boraginaceae } \\
\hline $\begin{array}{l}\text { Cynoglossum } \\
\text { zeylanicum } \\
\text { Thunb. Ex Lehm. }\end{array}$ & Chtkura & RKT 22969 & $\mathrm{H}$ & Rt & Dermatological—boils & $\mathrm{Hu}$ & $\mathrm{Da}$ & 54 & 0.54 & 54 & 54 & 0.54 \\
\hline \multicolumn{13}{|l|}{ Family: Brassicaceae } \\
\hline $\begin{array}{l}\text { Rephanus sativus } \\
\text { L. }\end{array}$ & Mooli & RKT 27049 & $\mathrm{H}$ & WP & Hepatic health cure-jaundice & $\mathrm{Hu}$ & Co & 87 & 0.87 & 87 & 87 & 0.87 \\
\hline \multicolumn{13}{|l|}{ Family: Cannabaceae } \\
\hline \multirow[t]{3}{*}{$\begin{array}{l}\text { Cannabis sativa } \\
\text { L. }\end{array}$} & Bhaang & GBPCSED3 & $\mathrm{H}$ & $\mathrm{Sd}, \mathrm{L}$ & $\begin{array}{l}\text { Gastrointestinal_-purgative and } \\
\text { laxative, carminative, constipation, } \\
\text { stomach ache }\end{array}$ & $\mathrm{Hu}$ & $\ln$ & 63 & 0.63 & 46 & 94 & 0.94 \\
\hline & & & & & Antidote-insect bite & & $\mathrm{Da}$ & & & 5 & & \\
\hline & & & & & Other-warm effect in winters & & $\ln , \mathrm{Co}$ & & & 43 & & \\
\hline
\end{tabular}


Table 1 Quantitative enumeration of ethnomedicinal plants used by marginal hill community of District Bageshwar (Continued)

\begin{tabular}{|c|c|c|c|c|c|c|c|c|c|c|c|c|}
\hline Botanical name & $\begin{array}{l}\text { Local } \\
\text { name }\end{array}$ & $\begin{array}{l}\text { Voucher/ } \\
\text { ident. no. }\end{array}$ & Habit & $\begin{array}{l}\text { Part } \\
\text { used }\end{array}$ & $\begin{array}{l}\text { Popular ailment uses (group and } \\
\text { categories) }\end{array}$ & $\begin{array}{l}\text { Used } \\
\text { in }\end{array}$ & Preparation & $F C^{a}$ & $\mathrm{RFC}^{\mathbf{b}}$ & $U R^{c}$ & $U R^{d}$ & $\mathrm{Cl}^{\mathrm{e}}$ \\
\hline \multicolumn{13}{|c|}{ Family: Caryophyllaceae } \\
\hline $\begin{array}{l}\text { Drymaria cordata } \\
\text { (L.) Willd. ex } \\
\text { Schult }\end{array}$ & -- & RKT 19989 & $\mathrm{H}$ & WP & Respiratory—cough & $\mathrm{Hu}$ & In & 19 & 0.19 & 7 & 7 & 0.07 \\
\hline \multirow{2}{*}{$\begin{array}{l}\text { Silene vulgaris } \\
\text { (Moench) Garcke }\end{array}$} & Pyankura & GBPCSED4 & $\mathrm{H}$ & WP & General health care-fever & $\mathrm{Hu}$ & De & 15 & 0.15 & 4 & 17 & 0.17 \\
\hline & & & & & $\begin{array}{l}\text { Gastrointestinal-removal of Ascaris } \\
\text { (antiparisitic) locally known as juga }\end{array}$ & & De & & & 13 & & \\
\hline
\end{tabular}

Family: Combretaceae

\begin{tabular}{|c|c|c|c|c|c|c|c|c|c|c|c|}
\hline $\begin{array}{l}\text { Terminalia } \\
\text { chebula } \\
\text { (Gaertner) Retz. }\end{array}$ & Harar & RKT 15469 & $\mathrm{~T}$ & $\mathrm{Fr}$ & $\begin{array}{l}\text { Gastrointestinal_-purgative and } \\
\text { laxative, carminative, constipation, } \\
\text { digestive problems, diarrhea }\end{array}$ & $\mathrm{Hu}$ & $\mathrm{PW}, \mathrm{Po}$ & 12 & 0.12 & 64 & 64 \\
\hline
\end{tabular}

Family: Cucurbitaceae

Momordica Karela

RKT $27529 \quad \mathrm{Cl} \quad \mathrm{Fr} \quad$ Circulatory-diabetes

$\mathrm{Hu} \quad \mathrm{Co}, \mathrm{In}$

$\begin{array}{lllll}39 & 0.39 & 39 & 39 & 0.39\end{array}$

Family: Dioscoreaceae

Dioscorea Genthi

deltoidea Wall.

RKT $27301 \mathrm{Cl} \quad \mathrm{Fr} \quad$ Respiratory—cough and cold

(Atu)

$\mathrm{Hu} \quad \mathrm{Co}$

$\begin{array}{lllll}32 & 0.32 & 32 & 32 & 0.32\end{array}$

Family: Ericaceae

Rhododendron Burans arboreum Smth

RKT $27288-T-F$

Hepatic health cure-liver complaints, Hu De tonic

$\begin{array}{lllll}47 & 0.47 & 64 & 64 & 0.64\end{array}$

Family: Euphorbiaceae

Emblica officinalis Aanwla Gaertn.

\section{RKT $21022 \quad \mathrm{~T} \quad \mathrm{Fr} \quad$ Circulatory-diabetes}

Euphorbia Dudhiya,

prolifera Ehrenb. Maikuri

Ex. Boiss

Family: Gentianceae

Swertia

angustifolia

Chiraita

Buch.-Ham. ex

D.Don

Family: Fabaceae

Merri

Microtyloma

uniflorum (Lam) Gahat/

Verdc.

L.

Trigonella

$$
\text { Maithi }
$$

foemun-graecum

L.

Vigna mungo L (Fabaceae)

Family: Lamiaceae

\begin{tabular}{|c|c|c|c|c|c|c|c|c|c|c|c|}
\hline Kala Bhatt & RKT 15664 & $\mathrm{H}$ & $\mathrm{Sd}$ & Hepatic health cure-jaundice & $\mathrm{Hu}$ & Co & 84 & 0.84 & 84 & 84 & 0.84 \\
\hline $\begin{array}{l}\text { Gahat/ } \\
\text { Kulthi }\end{array}$ & GBPCSED5 & $\mathrm{H}$ & $\mathrm{Sd}$ & Urinogenital disorder_stone & $\mathrm{Hu}$ & Co & 69 & 0.69 & 69 & 69 & 0.69 \\
\hline \multirow[t]{2}{*}{ Chalmoda } & RKT 26479 & $\mathrm{H}$ & L & General health care_-headache & $\mathrm{Hu}$ & Po & 44 & 0.44 & 18 & 22 & 0.22 \\
\hline & & & & $\begin{array}{l}\text { Dermatological_skin disease of } \\
\text { dogs-Luta }\end{array}$ & C & Po & & & 4 & & \\
\hline \multirow[t]{3}{*}{ Maithi } & RKT 28507 & $\mathrm{H}$ & $L, S d$ & Circulatory-diabetes & $\mathrm{Hu}$ & $\operatorname{lnf}$ & 31 & 0.31 & 11 & 61 & 0.61 \\
\hline & & & & $\begin{array}{l}\text { Gastrointestinal_carminative, obesity, } \\
\text { indigestion, constipation }\end{array}$ & & $\operatorname{lnf}$ & & & 47 & & \\
\hline & & & & Skeleton and muscles_-joint pain & & $\operatorname{lnf}$ & & & 3 & & \\
\hline Mass, Urad & RKT 27199 & $\mathrm{H}$ & Sd & Skeleton and muscles-fracture & $\mathrm{Hu}$ & $\ln$ & 61 & 0.61 & 61 & 61 & 0.61 \\
\hline
\end{tabular}

61
Gastrointestinal_-purgative and laxative, carminative, stomach ache

Respiratory-cough

Other-source of vitamin " $\mathrm{C}$ "

$\begin{array}{lllllll}\mathrm{Hu} & \text { In } & 35 & 0.35 & 8 & 85 & 0.85 \\ & \text { In } & & & 54 & & \\ & & & & & & \\ & \text { In } & & & 6 & & \\ & \text { In } & & & 17 & & \\ \text { I } & \text { Da } & 7 & 0.07 & 7 & 7 & 0.07\end{array}$

$\mathrm{Hu} \quad$ In

$\begin{array}{lllll}37 & 0.37 & 19 & 24 & 0.24\end{array}$

Dermatological—skin ailments 
Table 1 Quantitative enumeration of ethnomedicinal plants used by marginal hill community of District Bageshwar (Continued)

\begin{tabular}{|c|c|c|c|c|c|c|c|c|c|c|c|c|}
\hline Botanical name & $\begin{array}{l}\text { Local } \\
\text { name }\end{array}$ & $\begin{array}{l}\text { Voucher/ } \\
\text { ident. no. }\end{array}$ & Habit & $\begin{array}{l}\text { Part } \\
\text { used }\end{array}$ & $\begin{array}{l}\text { Popular ailment uses (group and } \\
\text { categories) }\end{array}$ & $\begin{array}{l}\text { Used } \\
\text { in }\end{array}$ & Preparation & $\mathrm{FC}^{\mathrm{a}}$ & $\mathrm{RFC}^{\mathbf{b}}$ & $U R^{c}$ & $U R^{d}$ & $\mathrm{Cl}^{\mathbf{e}}$ \\
\hline \multirow{3}{*}{$\begin{array}{l}\text { Ajuga bracteosa } \\
\text { Wall. ex Benth. }\end{array}$} & \multirow[t]{3}{*}{ Ratpatia } & \multirow[t]{3}{*}{ RKT 25182} & \multirow[t]{3}{*}{$\mathrm{H}$} & \multirow[t]{3}{*}{ WP } & General health care_-fever & \multirow[t]{3}{*}{$\mathrm{Hu}$} & \multirow[t]{3}{*}{ De } & \multirow[t]{3}{*}{55} & \multirow[t]{3}{*}{0.55} & 53 & \multirow[t]{3}{*}{72} & \multirow[t]{3}{*}{0.72} \\
\hline & & & & & Gastrointestinal—constipation & & & & & 16 & & \\
\hline & & & & & Urinogenital—diuretic & & & & & 3 & & \\
\hline \multirow[t]{3}{*}{$\begin{array}{l}\text { Ajuga parviflora } \\
\text { Benth. }\end{array}$} & \multirow[t]{3}{*}{ Ratpatia } & \multirow[t]{3}{*}{ RKT 26408} & \multirow[t]{3}{*}{$\mathrm{H}$} & \multirow[t]{3}{*}{ Rt } & $\begin{array}{l}\text { General health care-fever, throat } \\
\text { infection in animal (Galghotu) }\end{array}$ & $\begin{array}{l}\mathrm{Hu} \\
\text { and } \\
\mathrm{C}\end{array}$ & $\mathrm{De}, \mathrm{Em}$ & 56 & 0.56 & 58 & \multirow[t]{3}{*}{87} & \multirow[t]{3}{*}{0.87} \\
\hline & & & & & $\begin{array}{l}\text { Gastrointestinal-constipation, } \\
\text { stomach ache }\end{array}$ & $\mathrm{Hu}$ & De, In & & & 25 & & \\
\hline & & & & & Urinogenital—stone & & De & & & 4 & & \\
\hline $\begin{array}{l}\text { Leucas lanata } \\
\text { Benth }\end{array}$ & Nirasi Jhad & RKT 29214 & $\mathrm{H}$ & L & Respiratory—cough & $\mathrm{Hu}$ & De & 80 & 0.8 & 80 & 80 & 0.8 \\
\hline $\begin{array}{l}\text { Mentha arvensis } \\
\mathrm{L} \text {. }\end{array}$ & Pudina & RKT 4355 & $\mathrm{H}$ & L & $\begin{array}{l}\text { Gastrointestinal_-stomach ache, } \\
\text { vomiting }\end{array}$ & $\mathrm{Hu}$ & De & 43 & 0.43 & 50 & 50 & 0.5 \\
\hline $\begin{array}{l}\text { Micromeria } \\
\text { biflora Benth. }\end{array}$ & -- & RKT 22949 & $\mathrm{H}$ & WP & General health care-fever & $\mathrm{Hu}$ & De & 6 & 0.06 & 6 & 6 & 0.06 \\
\hline \multirow{3}{*}{$\begin{array}{l}\text { Ocimum } \\
\text { basilicum L. }\end{array}$} & \multirow[t]{3}{*}{ Tulsi } & \multirow[t]{3}{*}{ RKT 19325} & \multirow[t]{3}{*}{ S } & \multirow[t]{3}{*}{$\mathrm{L}, \mathrm{Sd}$} & General health care-fever & $\mathrm{Hu}$ & De & \multirow[t]{3}{*}{88} & 0.88 & 33 & \multirow[t]{3}{*}{97} & 0.97 \\
\hline & & & & & Respiratory—cough and cold & & De & & & 41 & & \\
\hline & & & & & $\begin{array}{l}\text { Other-herbal tea, warm effect in } \\
\text { winters }\end{array}$ & & De & & & 23 & & \\
\hline Origanum & Van Tulsi & RKT 29244 & & $L, R t$ & General health care-fever & $\mathrm{Hu}$ & De & 31 & 0.31 & 15 & 71 & 0.71 \\
\hline & & & & & Respiratory_cough and cold & & De & & & 18 & & \\
\hline & & & & & Dermatological—wounds & & $\mathrm{Em}$ & & & 29 & & \\
\hline & & & & & Other_herbal tea & & $\operatorname{lnf}$ & & & 9 & & \\
\hline $\begin{array}{l}\text { Thymus serpyllum } \\
\text { L. }\end{array}$ & $\begin{array}{l}\text { Van- } \\
\text { ajwayan }\end{array}$ & RKT 27966 & $\mathrm{H}$ & WP & Skeleton and muscles_-joint pain & $\mathrm{Hu}$ & $\mathrm{Em}$ & 18 & 0.18 & 3 & 14 & 0.14 \\
\hline & & & & & Respiratory—asthma & & $\mathrm{Em}$ & & & 3 & & \\
\hline & & & & & $\begin{array}{l}\text { Gastrointestinal_digestive and } \\
\text { stomach problems }\end{array}$ & & $\mathrm{Em}$ & & & 4 & & \\
\hline & & & & & Other—spices and condiments & & $\mathrm{Da}$ & & & 4 & & \\
\hline Family: Liliaceae & & & & & & & & & & & & \\
\hline Asparagus & Keruwa & RKT 28055 & S & Rt & Immuno-regulatory—stimulant & $\mathrm{Hu}$ & Pw & 46 & 0.46 & 15 & 65 & 0.65 \\
\hline & & & & & Hepatic health cure-tonic & & Pw & & & 39 & & \\
\hline & & & & & Gastrointestinal_-stomach ache & & De & & & 11 & & \\
\hline Polygonatum & Maha- & RKT 26144 & $\mathrm{H}$ & WP & Hepatic health cure-tonic & $\mathrm{Hu}$ & De & 21 & 0.21 & 13 & 34 & 0.34 \\
\hline $\begin{array}{l}\text { IIrnitollum } \\
\text { (Wall.) Royle }\end{array}$ & Meda & & & & Dermatological—cuts and wounds & & Po & & & 14 & & \\
\hline & & & & & Circulatory_blood purifier & & Co & & & 7 & & \\
\hline Polygonatum & Meda & RKT 25894 & $\mathrm{H}$ & Rt & Gastrointestinal_carminative & $\mathrm{Hu}$ & $\ln$ & 15 & 0.15 & 8 & 19 & 0.19 \\
\hline verticillatum L. & & & & & Dermatological—wounds & & Po & & & 11 & & \\
\hline Family: Moraceae & & & & & & & & & & & & \\
\hline $\begin{array}{l}\text { Ficus palmata } \\
\text { Forsk. }\end{array}$ & Bedu & RKT 28094 & $\mathrm{~T}$ & $L t$ & Dermatological—cuts and wounds & $\mathrm{Hu}$ & $\mathrm{Da}$ & 48 & 0.48 & 39 & 39 & 0.39 \\
\hline Ficus roxburghii & Timul & GBPCSED6 & $\mathrm{T}$ & $\mathrm{Fr}$ & Gastrointestinal_acidity, carminative & $\mathrm{Hu}$ & Co & 26 & 0.26 & 45 & 48 & 0.48 \\
\hline & & & & & Circulatory_blood pressure & & Co & & 0 & 3 & & 0 \\
\hline Family: Myricaceae & & & & & & & & & & & & \\
\hline Psidium guajava & Amrood & GBPCSED7 & $\mathrm{T}$ & L & General health care-mouth blisters & $\mathrm{Hu}$ & $\ln$ & 12 & 0.12 & 12 & 12 & 0.12 \\
\hline
\end{tabular}


Table 1 Quantitative enumeration of ethnomedicinal plants used by marginal hill community of District Bageshwar (Continued)

Botanical name Local Voucher/ Habit Part Popular ailment uses (group and Used Preparation $\mathrm{FC}^{\mathbf{a}} \mathrm{RFC}^{\mathbf{b}} \quad \mathrm{UR}^{\mathbf{c}} \mathrm{UR}^{\mathbf{d}} \mathrm{Cl}^{\mathbf{e}}$

name ident. no. used categories)

L.

Family: Orchidaceae

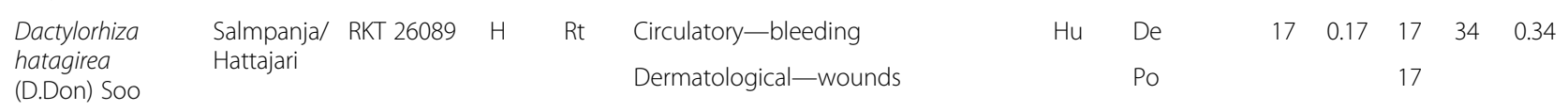

Family: Plantaginaceae

\begin{tabular}{|c|c|c|c|c|c|c|c|c|c|c|c|c|}
\hline $\begin{array}{l}\text { Plantago ovate } \\
\text { Forsk. }\end{array}$ & Isabgoal & RKT 1899 & $\mathrm{H}$ & $\mathrm{Sd}$ & $\begin{array}{l}\text { Gastrointestinal-constipation, } \\
\text { digestive problems, diarrhea }\end{array}$ & $\mathrm{Hu}$ & $\ln$ & 74 & 0.74 & 83 & 83 & 1.83 \\
\hline $\begin{array}{l}\text { Plantago } \\
\text { lanceolata L. }\end{array}$ & Jonkpuri & RKT 8154 & $\mathrm{H}$ & Rt & $\begin{array}{l}\text { Gastrointestinal_-removal of stomach } \\
\text { worm of domestic animals }\end{array}$ & C & $\ln$ & 43 & 0.43 & 43 & 43 & 0.43 \\
\hline
\end{tabular}

Family: Poaceae

\begin{tabular}{|c|c|c|c|c|c|c|c|c|c|c|c|c|}
\hline \multirow[t]{2}{*}{$\begin{array}{l}\text { Hordium vulgare } \\
\mathrm{L} \text {. }\end{array}$} & Jau & RKT 26630 & $\mathrm{H}$ & Sd & $\begin{array}{l}\text { Hepatic health cure-warm and } \\
\text { nutritive effect }\end{array}$ & $\mathrm{Hu}$ & Co & 46 & 0.46 & 46 & 63 & 0.63 \\
\hline & & & & & Dermatological—burns & & $\mathrm{O}$ & & & 17 & & \\
\hline Setaria italica L. & Kouni & RKT 7389 & $\mathrm{H}$ & $\mathrm{Sd}$ & $\begin{array}{l}\text { Dermatological_measles and chicken } \\
\text { pox }\end{array}$ & $\mathrm{Hu}$ & $\mathrm{Co}$ & 91 & 0.91 & 91 & 91 & 0.91 \\
\hline
\end{tabular}

Family: Podophyllaceae

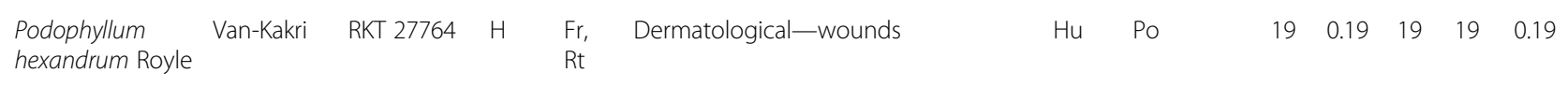

Family: Polygonaceae

Rheum emodi Dolu
Wall.

RKT $27793 \quad H \quad$ Rt General health care-fever

$\mathrm{Hu} \quad \mathrm{De}$

$\begin{array}{lllll}31 & 0.31 & 15 & 42 & 0.42\end{array}$

Dermatological—wounds

Po

27

Family: Punicaceae

Punica granatum Darim $\mathrm{L}$.

RKT 28845 T $\quad$ Fr Respiratory—cough and cold

$\mathrm{Hu} \quad \mathrm{In}$

$\begin{array}{llll}59 & 0.59 & 49 & 71\end{array}$

Hepatic health cure-anemia

De

10

Other-source of vitamin " $\mathrm{C}$ "

De, In

12

Family: Ranunculaceae

$\begin{array}{ll}\begin{array}{l}\text { Aconitum } \\ \text { heterophyllum }\end{array} & \text { Atis } \\ \text { Wall. } & \\ \begin{array}{l}\text { Ranunculus } \\ \text { repens L. }\end{array} & \text { Aingadua } \\ & \\ & \\ \text { Thalictrum } & \text { Uppankat } \\ \text { foliosum DC. } & \text { hi/Mamira } \\ & \\ \text { Family: Rosaceae } & \\ \begin{array}{l}\text { Duchesnea indica } \\ \text { (Andrews) Focke }\end{array} & \\ \begin{array}{l}\text { Prunus persica } \\ \text { Stokes. }\end{array} & \text { Aaru } \\ \begin{array}{l}\text { Rosa moschata } \\ \text { Hermm. }\end{array} & \text { Kunja } \\ & \\ \begin{array}{l}\text { Rubus ellipticus } \\ \text { Smith. }\end{array} & \text { Hisalu }\end{array}$

RKT $29008 \quad H \quad$ Rt $\quad$ General health care-fever
Gastrointestinal-vomiting

GBPCSED8 H Rt Dermatological-boils

Gastrointestinal-intestinal pains (NasPalatana)

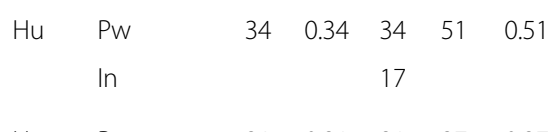

$\begin{array}{lllll}21 & 0.21 & 21 & 27 & 0.27\end{array}$

RKT $29204 \quad H \quad$ WP Ophthalmic —eye infection (white dot-cataract)

Other-insect repellent

$\ln$

6

$\begin{array}{lllll}4 & 0.04 & 9 & 21 & 0.21\end{array}$

GBPCSED9 $H \quad L \quad$ Dermatological—burns and removal $\quad H u \quad P o$ of burn scars

RKT $26465 T \quad$ L $\quad$ General health care-Headache $\quad H u \quad$ Po

RKT 28695 S L, F Dermatological—cuts and wounds, Hu Po boils

Ophthalmic —eye diseases

Ste

$\mathrm{Hu}$ De

De

12 
Table 1 Quantitative enumeration of ethnomedicinal plants used by marginal hill community of District Bageshwar (Continued)

\begin{tabular}{|c|c|c|c|c|c|c|c|c|c|c|c|c|}
\hline Botanical name & $\begin{array}{l}\text { Local } \\
\text { name }\end{array}$ & $\begin{array}{l}\text { Voucher/ } \\
\text { ident. no. }\end{array}$ & Habit & $\begin{array}{l}\text { Part } \\
\text { used }\end{array}$ & $\begin{array}{l}\text { Popular ailment uses (group and } \\
\text { categories) }\end{array}$ & $\begin{array}{l}\text { Used } \\
\text { in }\end{array}$ & Preparation & $\mathrm{FC}^{\mathbf{a}}$ & $\mathrm{RFC}^{\mathbf{b}}$ & $U R^{c}$ & $U R^{d}$ & $\mathrm{Cl}^{\mathbf{e}}$ \\
\hline \multicolumn{13}{|l|}{ Family: Rubiaceae } \\
\hline $\begin{array}{l}\text { Rubia cordifolia } \\
\text { L. }\end{array}$ & Manjistha & RKT 27933 & $\mathrm{H}$ & Rt & General health care-fever & $\mathrm{Hu}$ & De & 27 & 0.27 & 23 & 23 & 0.23 \\
\hline \multicolumn{13}{|l|}{ Family: Rutaceae } \\
\hline \multirow{4}{*}{$\begin{array}{l}\text { Citrus aurantifolia } \\
\text { (Christm) Sw. }\end{array}$} & Kagji & GBPCSED10 & $\mathrm{T}$ & $\mathrm{Fr}$ & General health care-headache & $\mathrm{Hu}$ & De & 38 & 0.38 & 20 & 94 & 0.94 \\
\hline & Nimboo & & & & $\begin{array}{l}\text { Gastrointestinal—constipation, weight } \\
\text { loss }\end{array}$ & & De & & & 23 & & \\
\hline & & & & & Respiratory—cold & & De & & & 19 & & \\
\hline & & & & & $\begin{array}{l}\text { Other-herbal tea, source of vitamin } \\
\text { " } C \text { " }\end{array}$ & & De & & & 32 & & \\
\hline \multirow[t]{3}{*}{ Citrus hystrix DC. } & $\begin{array}{l}\text { Jamer/ } \\
\text { Jamir }\end{array}$ & GBPCSED11 & $\mathrm{T}$ & $\mathrm{Fr}$ & $\begin{array}{l}\text { Gastrointestinal—removal of Ascaris } \\
\text { (antiparisitic) locally known as juga }\end{array}$ & $\mathrm{Hu}$ & $\ln$ & 38 & 0.38 & 27 & 50 & 0.5 \\
\hline & & & & & Respiratory—cold & & $\ln$ & & & 7 & & \\
\hline & & & & & Antidote-against poison & C & $\mathrm{Em}$ & & & 16 & & \\
\hline \multirow{4}{*}{$\begin{array}{l}\text { Zanthoxylum } \\
\text { armatum DC }\end{array}$} & Timoor/ & RKT 28615 & S & Sd & General health care-toothache & $\mathrm{Hu}$ & $\ln$ & 61 & 0.61 & 21 & 77 & 0.77 \\
\hline & 11 & & & & Respiratory_cough and cold & & $\ln$ & & & 19 & & \\
\hline & & & & & Gastrointestinal_carminative & & $\ln$ & & & 6 & & \\
\hline & & & & & Other-spices and condiments & & $\ln$ & & & 31 & & \\
\hline
\end{tabular}

Family: Saxifragaceae

Bergenia ciliata Silphora (Haw) Sternb

Family: Scorphulariaceae

Picrorhiza Kutk kurrooa Royle.

RKT $27765 \quad H \quad$ Rt General health care-fever

Gastrointestinal—abdominal pain

Verbascum

Akalveer

thapsus L.

Family: Urticaceae

Urtica dioica $\mathrm{L}$.

Shishun/
Bichhu
ghas

RKT $22903 \quad S$

ghas

Family: Violaceae

Viola betonicifolia Garurjadi/ J.E. Smith garurabuti

Viola canescens

Wall. Ex Roxb

Family: Zingiberaceae

Curcuma longa Haldi

L.

RKT 5970

GBPCSED12 H WP Antidote-snake bite

RKT $17561 \quad H \quad$ WP

Other-to increase lactation in

milching animals

Other-to increase lactation in milching animals

Skeleton and muscles-joint pain

Hepatic health cure-warm and nutritive effect

$\begin{array}{ll}\mathrm{Hu} & \mathrm{Da} \\ \mathrm{Hu} & \mathrm{Co}\end{array}$

$\begin{array}{lllll}37 & 0.37 & 31 & 52 & 0.52\end{array}$

$\mathrm{Hu}$
$\mathrm{C}$
$\mathrm{Po}$

$\begin{array}{llll}12 & 0.12 & 13 & 13\end{array}$

$\begin{array}{lllll}29 & 0.29 & 29 & 29 & 0.29\end{array}$

General health care-internal injury $\mathrm{Hu}$ De

Dermatological—cuts and wounds, cosmetics

Respiratory—cough

Hedychium spicatum Buch.

Van Haldi RKT 24059 H $\quad$ Rh

Ham. ex Smith. $\begin{array}{lllll}78 & 0.78 & 39 & 91 & 0.91\end{array}$

Da $\quad 36$

De 16

$\begin{array}{lllll}13 & 0.13 & 30 & 52 & 0.52\end{array}$
Gastrointestinal_-intestinal problems, $\mathrm{Hu} \quad$ Pw purgative and laxative, carminative

Respiratory—cough

PW

Dermatological—cosmetics, anti-lice Hu \& PW 
Table 1 Quantitative enumeration of ethnomedicinal plants used by marginal hill community of District Bageshwar (Continued)

\begin{tabular}{|c|c|c|c|c|c|c|c|c|c|c|c|c|}
\hline Botanical name & $\begin{array}{l}\text { Local } \\
\text { name }\end{array}$ & $\begin{array}{l}\text { Voucher/ } \\
\text { ident. no. }\end{array}$ & Habit & $\begin{array}{l}\text { Part } \\
\text { used }\end{array}$ & $\begin{array}{l}\text { Popular ailment uses (group and } \\
\text { categories) }\end{array}$ & $\begin{array}{l}\text { Used } \\
\text { in }\end{array}$ & Preparation & $\mathrm{FC}^{\mathrm{a}}$ & $\mathrm{RFC}^{\mathbf{b}}$ & $U R^{c}$ & $U R^{d}$ & $\mathrm{Cl}^{\mathbf{e}}$ \\
\hline & & & & & & $C$ & & & & & & \\
\hline $\begin{array}{l}\text { Zingiber officinale } \\
\text { Rosc. }\end{array}$ & Adrak & RKT 5921 & $\mathrm{H}$ & $\mathrm{Rh}$ & Respiratory_Cough and cold & $\mathrm{Hu}$ & $\mathrm{Em}$ & 89 & 0.89 & 89 & 89 & 0. \\
\hline
\end{tabular}

Atu aerial tuber, $B$ bulb, $C$ cattle, $C l$ climber, Co cooking, De decoction, Da direct application, Em emulsion, $F$ flower, Fr fruit, $H$ herb, $I$ insect, Inf infusion, In ingestion, $h r$ hour, Hu human, $L$ leaves, $L t$ latex, $O$ ointment, Po poultice, $P w$ powder, $R h$ rhizome, Rt root, $S$ shrub, Sd seed, Ste steam, $T$ tree, WP whole plant a Use citation of taxa (the no. of informants that referred the taxon)

${ }^{\mathrm{b}} \mathrm{RFC}=\mathrm{FC} / N$, where $N$ is the total no. of informants

'Use reports of the taxon by ailment category

dUse reports of the taxon

${ }^{\mathrm{e}} \mathrm{Cl}=\mathrm{UR} / \mathrm{N}_{\mathrm{t}}$, where $N_{\mathrm{t}}$ is the total no. of reported taxa

potential bioactive compounds after heating [26]. The pleasant taste of the herbal drug can be attuned by adding together honey or sugar [27]. Ingestion and poultice were also common after crushing and/or mixing the plant parts with some solvent for application as paste and Band-Aid. In skeletal, muscle, and dermatological issues, application of plant parts as ointment was most prevalent.

The community and Vaidyas identify each medicinal plant with a specific vernacular name. For example, Bergenia ciliata is identified by the community with a local name "Pattharchatta" (stone destroyer), and it is used in curing kidney stones. Plantago ovate is called "Jonkpuri" (jonk resembles worms) and is used in the treatment of Ascaris and other worms. Viola betonicifolia named "Garur-Jadi" (Garur means eagle), and it is used as an antidote to treat snake bites. Commonly, the community identifies a native name for species based on its local uses, ecology, physiology, anatomy, pharmacological activity, etc. [28].

It was recorded that the species were used to cure a total of 12 major ailments (Fig. 6). Most species were used for curing gastrointestinal and general health disorders. It was followed by species used for treating dermatological and respiratory problems.

Lamiaceae has been the most dominating family for ethnomedicinal uses in the trans-Himalayan zone of
Nepal [29] and Garhwal Himalaya in India as well [30]. Although the people in remote areas are still dependent on the traditional herbal cure system, it is being practiced by a few elderly people only. The young generation is not interested to take up this profession given minimal profit $[3,10,12]$. The common plant parts used in the present study are similar to other investigations [31-35]. The roots being the storage part of the plant contain valuable bioactive compounds [36]. Apart from the root part, leaves also contain a high concentration of healthbeneficial secondary metabolites, phytochemicals, and essential oils, which contribute significantly to phototherapy or treatment of various health disorders [37-40]. The study reports $60 \%$ more species than reported earlier for the area under investigation [41-45].

\section{Quantitative analysis of ethnomedicinal information}

The use value of important ethnomedicinal species was also calculated to depict the number of uses reported by the informants related to the utility of a species for a specific ailment or different ailments (Tables 1 and 3). Two forms of use reports were analyzed; the URc defines the use of a particular species to cure specific ailments as reported by all the informants, while URd reports the sum of all the uses for a particular disease/

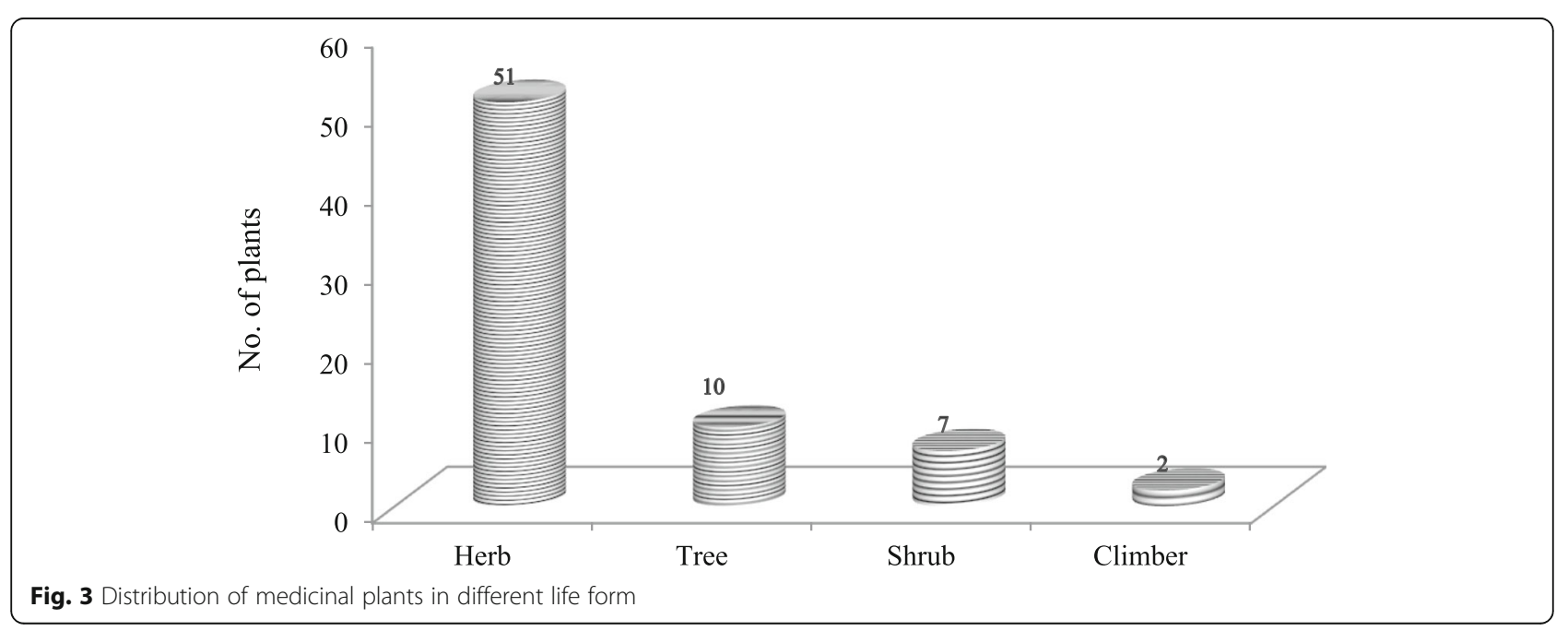




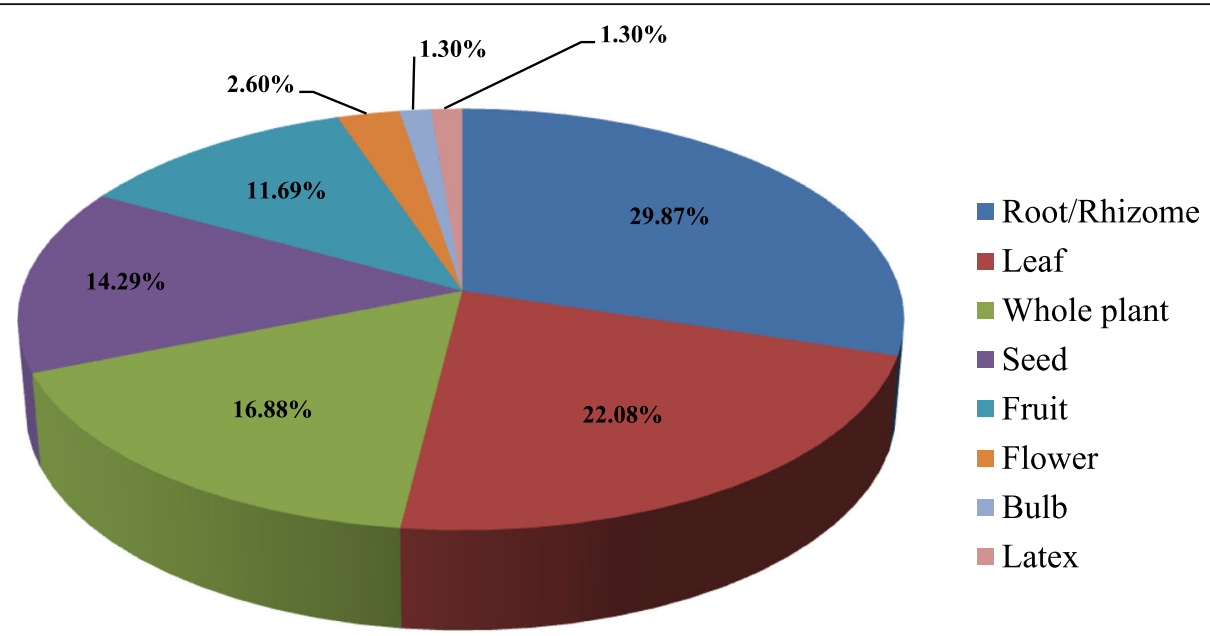

Fig. 4 Plant part used in preparation of medicine

ailment. Ocimum basilicum, Cannabis sativa, Citrus aurantifolia, Curcuma longa, and Setaria italica have been top positioned in terms of use-reports and different ailments cured.

The usefulness of a species can be represented through its RFC value, which ranged 0.03 to 0.91 for different species (Table 1). Species with maximum RFC value were Setaria italica, Zingiber officinale, Ocimum basilicum, and Raphanus sativus which depict their higher use, while those with the least value comprised Duchesnea indica and Thalictrum foliosum.

The cultural importance index (CIs) specifies the distribution and importance of species in traditional herbal system, and the value ranged from 0.03 to 0.97 . A total of 21 species have been identified as the most commonly used (Table 3). Ocimum basilicum, Cannabis sativa, and Citrus aurantifolia registered the highest cultural importance in the traditional herbal cure system. Low CI values specify that these species are either least used, or their use is declining in traditional herbal cure system [46].

An analysis of the informant consensus factor (Fic) for 12 broad treatment categories ranged between 0.92 and 1.0 (Table 4). The data revealed high homogeneity as per local people for all treatments. The immuno-regulatory category was assigned the value 1 due to the presence of only one taxon in the particular category. Apart from this, hepatic health care and urogenital categories obtained the value of 0.98 indicating well-defined criteria among the local population and non-random selection of species for the ailment category. Asparagus recemosus, Glycine max, Hordeum vulgare, Polygonatum cirrhifolium, Punica granatum, Raphanus sativus, and Urtica dioica not only used in hepatic health care but also provide nutritive benefits and warm potency, particularly at higher altitude areas. These species are commonly used in the daily food habit of the local community. Also, a higher value of Fic verifies the distribution of the different species used for a specific ailment. The urogenital category, with only 4 taxa included, comes second in terms of CI as there is a widely accepted notion of using these species for such disorders. The higher value of informant consensus factor for all the ailment categories also implies that the documented species are the most commonly used in traditional healing system.

The gastrointestinal ailments comprised of 695 use reports from the total categories with a medicinal importance index value of 30.22 (Table 4). Some most sought species in this category are Cannabis sativa, Citrus aurantifolia, Angelica galuca, Ajuga parviflora, and Emblica officinalis. These species are placed following their use reports mentioned during data collection. In the category of general health care, 22 species are being used with 524 numbers of use-reports and medical importance of 23.82. The species indicated with the highest number of use-reports are Ocimum basilicum, Citrus aurantifolia, Curcuma longa, Ajuga parviflora, and Picrorhiza kurrooa based on user reports. The dermatological category ranks third with 21 taxa in use and a use-report value of 617 and medicinal importance of 29.82. The main species employed for this category based on the use reports are Setaria italica, Eupatorium adenophorum, and Artemisia martima. Although the hepatic health cure category comprised of only 8 taxa, it has a medicinal importance index value of 45.50 , which is highest of all the categories since the species used under the category are of daily usage and are often included in daily food products with nutritive values. The species include Glycine max, Hordeum vulgare, Punica granatum, Urtica dioica, Polygonatum cirrhifolium, etc. In other works carried out in Uttarakhand, they have reported these medicinal plants and use different plant parts in a different ratio to cure disease or aliments [16, 30, 31, 41-43, 45, 47-49]. 
Table 2 Bio-processing of medicinal plants of District Bageshwar

\begin{tabular}{ll}
\hline Scientific name & Mode of administration \\
\hline Aconitum heterophyllum Wall. & $\begin{array}{l}\text { Dry root powder (1 TS) taken orally with boiled water twice a day for 2-3 days against fever; 1-2 roots chewed } \\
\text { to control vomiting. }\end{array}$ \\
Acorus calamus L. & $\begin{array}{l}\text { Root powder mixed with grains used as insect repellent; } 3-4 \text { dry roots heated with mustard oil applied on the } \\
\text { sprain and inflammatory region. }\end{array}$
\end{tabular}

Ageratina adenophora (Spreng.) L Leaf paste prepared from $100 \mathrm{~g}$ fresh leaf twigs applied on affected parts for early healing.

King \& H. Rob

Ajuga bracteosa Wall. ex Benth. Juice of whole plant (10-20 ml) taken twice a day for 2-3 days.

Ajuga parviflora Benth.

Decoction prepared from $100 \mathrm{~g}$ fresh or dried roots with water given 3-5 TS orally in fever, stomach ache, and constipation for 5 days; this decoction taken orally in empty stomach regularly for stone; $1-2$ leaves chewed on empty stomach for gastric problem; decoction of whole plant (5-8) crushed with red chili (3) and $100 \mathrm{~g}$ Jiggery (Gur) given twice a day for $2-3$ days to treat throat infection in domestic animals.

Allium sativum $\mathrm{L}$.

Paste prepared from 5-7 spilled bulb heated with $20 \mathrm{ml}$ mustard oil, massage on joints.

Angelica glauca Edgew.

Root powder $(50 \mathrm{~g}$ ) mixed with $100 \mathrm{ml}$ water used to control vomiting and stomach ache; rhizomes are used as spices and condiments and tea (as flavor).

Artemisia martima L.

Juice (5-10 $\mathrm{ml}$ ) of fresh leaf applied on the affected area.

Asparagus racemosus Willd.

Root decoction $(100 \mathrm{~g})$ prepared in water given to cure stomach ache ( $5 \mathrm{ml}$ for adult, 1 TS for children) for 3-5 days, one palm full root powder taken with water as stimulant and tonic.

Berberis asiatica Roxb. ex DC

Root powder (100-150 g) taken with warm water given twice a day for 3 days against fever; fresh or dried roots soaked in water overnight, filtered, and taken orally to cure diabetes in empty stomach.

Bergenia ciliata (Haw) Sternb.

Fresh or dried roots (50-100 g) socked overnight and filtered, taken orally in morning for kidney stone. Root powder $(50 \mathrm{~g})$ taken with water twice a day for urinary infection.

Cannabis sativa L.

Grinded seeds cooked with some local vegetables (e.g., Colacasia esculanta, Brassica oleracea) for warm effect; broiled seeds are grinded with salt and green chili to prepare salt (Pahadi namak). Broiled seeds grinded with Punica garnatum mixed with green leaves of coriandum, green chili, salt, and sugar to prepare Chatni; fresh leaves crushed with 3-5 seeds of black pepper and applied on insect bite.

Centella asiatica $\mathrm{L}$.

Fresh leaf paste is applied on forehead.

Citrus aurantifolia (Christm) Sw.

Juice extracted from fruit mixed with 1 TS honey, and $50 \mathrm{ml}$ water taken orally in empty stomach for constipation and weigh loss; lemon tea used in fever and cold.

Citrus hystrix DC.

Fruit juice given orally (1 TS) to children for removal of Ascaris; cough and cold $10 \mathrm{ml}$ thrice a day; fruit juice with mentha leaves $(100 \mathrm{~g})$ and coriander seeds made into paste given to domestic animals against poison.

Coriandrum sativum $\mathrm{L}$. Seed (80-100 g) paste mixed with 1-2 I processed curd (Mattha) is given to domestic animals against poison for 2-3 days.

Curcuma longa $\mathrm{L}$. Haldi powder ( $5 \mathrm{~g}$ ) mixed with a full glass of warm milk for internal injury; paste of rhizome applied on cuts and wounds.

Cynoglossum zeylanicum Thunb. Ex Fresh or dried root paste applied on the affected parts.

Lehm.

Dactylorhiza hatagirea (D.Don) Soo.

Dioscorea bulbifera $\mathrm{L}$.

Drymaria cordata (L.)

Duchesnea indica (Andrews) Focke

Emblica officinalis Gaertn.

Euphorbia sp.

Ficus palmata Forsk.

Ficus roxburghii Wall.

Glycine max (L.) Merri

Hedychium spicatum Buch. Ham. ex Smith.

Hordium vulgare $L$.
Decoction of $100 \mathrm{~g}$ root with water taken orally $(10-15 \mathrm{ml})$ twice a day for excessive bleeding; root paste applied on wounds.

Broiled fruit and cooked vegetable.

Juice of aerial parts (2-4 drops) taken orally for 2-3 days.

Leaf paste is regularly applied on affected part.

Fresh fruits are chewed regularly to control diabetes; dried fruits (3-5) boiled with water, filtered, and taken orally against cough and stomach ache; fresh and processed fruits are source of vitamin "C."

Whole plant (50-100) mixed with FYM.

Milky latex applied on cuts and wounds.

Fresh fruits are cooked as vegetable.

Bhatt ka Jaula (an indigenous dish) is prepared from paste of seeds (soaked overnight) and cooked with rice in an iron vessel Kadahi.

Dried rhizome powder (2-3 g) taken with hot water once a day; paste of fresh rhizome used as anti-lice.

Sattu prepared from $200 \mathrm{~g}$ broiled seeds mixed with $100 \mathrm{~g}$ jaggery (Gur) and $100 \mathrm{~g}$ Ghee for warm and nutritive effect; $50 \mathrm{~g}$ broiled seeds heated with $40 \mathrm{ml}$ mustard oil applied on burns. 
Table 2 Bio-processing of medicinal plants of District Bageshwar (Continued)

Scientific name
Leucas lanata Benth
Mentha arvensis L.
Micromeria biflora Benth.
Macrotyloma uniflorum (Lam)
Verdc.
Momordica charanti L.
Ocimum basilicum L.
Origanum vulgare L.
Picrorhiza kurrooa Royle.
Plantago ovate Forsk.
Plantego lanceolata L.
Podophyllum hexandrum Royle
Polygonatum cirrhifolium (Wall.)
Royle
Polygonatum verticillatum L. All
Prunus persica Stokes.
Psidium guajava L.
Punica granatum L.

Ranunculus repens $\mathrm{L}$.

Rephanus sativus L.

Rheum emodi Wall.

Rhododendron arboreum Smth

Rosa moschata Hermm.

Rubia cordifolia L.

Rubus ellipticus Smith.

Saussurea costus (Falc.) Lipsch

Setaria italica L.

Silene vulgaris (Moench) Garcke

Swertia spp.

Taraxacum officinale Weber.

Tegetus erecta L.

Terminalia chebula (Gaertner) Retz.

Thalictrum foliosum DC.

Thymus serpyllum $\mathrm{L}$.

Mode of administration

Leaf juice with 3-5 drops of breast milk taken orally twice a day for 1 week.

Leaves $(100 \mathrm{~g})$ boiled with water and filter, the filtrate $(50 \mathrm{ml})$ given orally twice a day.

Juice of whole plant with water (1-2 times in a day).

Gahat ka Ras (an indigenous dish) prepared by $150 \mathrm{~g}$ seeds cooked with water (1 I) until the volume reduced $(100 \mathrm{ml})$ and taken regularly.

Vegetable and juice $(50 \mathrm{ml})$ of fresh fruit taken regularly.

Decoction of $100 \mathrm{~g}$ leaves and seeds, zinger $(50 \mathrm{~g}), 5$ seeds black paper with $150 \mathrm{ml}$ water taken orally 2-3 times a day for fever, cough, and cold; aerial part used to make herbal tea.

Decoction of $100 \mathrm{~g}$ fresh and dried leaves with water taken orally $(10 \mathrm{ml})$ for a week in cough, cold, and fever; root paste applied on wounds.

Decoction of $50 \mathrm{~g}$ root with water taken orally against fever and abdominal pain for 5-7 days.

Seeds $(10 \mathrm{~g})$ soaked overnight or consumed directly with water twice a day for 30 days against constipation and digestive problems; Isabgoal (15 g) mixed with 10 TS fresh curd taken after meal for diarrhea.

Paste of roots $(100 \mathrm{~g})$ given to domestic animals.

Root paste applied on wound.

Small pieces of tuber (8-10) soaked in water for overnight, taken in empty stomach for weakness, and develop immunity; cooked green leaves eaten as blood purifier; root paste applied on cuts and wounds.

Root powder $(50 \mathrm{~g})$ is taken with warm water in gastric complaints; fresh root paste applied for wound healing.

Fresh leaf paste applied on head for $2-3 \mathrm{~h}$.

Fresh leaves are chewed.

Powder (50 g) of dried fruit peel taken orally with warm water for old cough; fruit juice $(50 \mathrm{ml})$ given twice a day to anemic patient.

Root paste $(50 \mathrm{~g}$ ) applied for boils, and $30-50 \mathrm{ml}$ filtered root extract (juice) is given twice a day against intestinal pain.

Vegetable prepared from fresh leaves and root as salad.

Decoction of $100 \mathrm{~g}$ root with warm water taken orally $(10 \mathrm{ml})$ for fever twice a day; root paste applied on wounds.

Juice extracted from fresh flowers

Fresh leaf paste is applied on cuts, wounds, and boils; water extracted from fresh flowers used in eye diseases.

Root decoction with water given orally (1-2 TS) against fever twice a day to children ( 5 months-10 years)

Decoction $(10 \mathrm{ml})$ of $100 \mathrm{~g}$ roots with water taken orally against fever and stomach ache for 5 days.

Decoction of root $(50 \mathrm{~g})$ with water given against dysentery for 3-5 days twice a day; root powder $(50 \mathrm{~g})$ taken orally with boiled water in fever, cough, and stomach ache.

Koni ka Jaula (an indigenous dish) prepared from seeds cooked with water.

Root decoction $(10 \mathrm{ml})$ with warm water given against fever for 3 days; 1 TS is used for removal of Ascaris (Juga); leaves are used as a vegetable.

Juice of fresh leaves (100 g) given with boiled water 3 TS for 3-5 days for fever; Panchang (whole plant) is used after soaking overnight and taken (50-100 ml) orally in empty stomach for 15 days.

For snake bite: juice of whole plant with water taken orally (1-2 TS) thrice a day and applied on injured part for 1 week; mixture of $100 \mathrm{~g}$ roots with 9 seeds of black pepper, 1-2I processed curd (Mattha), and $250 \mathrm{~g}$ paste of black soybean given to increase lactation in milching animals.

Fresh leaf juice with water taken against fever (3-5 TS twice a day); leaf extract (2-3 drops) in ear infection; fresh leaf paste is applied for healing cuts and wounds.

Dried fruit powder $(100 \mathrm{~g}$ ) given orally with boiled water twice a day for $3-5$ days in stomach ache; dried fruit crushed with water and given (1-2 ml) orally to children ( 3 months to 5 years) and small amount applied around the navel.

Fresh roots $(50 \mathrm{~g})$ soaked in rose water $(100 \mathrm{ml})$ for overnight, filtered, and used as eye drop.

Paste of whole plant mixed with mustard oil gently applied on joints; whole plants juice $(10 \mathrm{ml})$ mixed with honey $(20 \mathrm{~g})$ is taken orally for cough and asthma; broiled seeds $(10-15 \mathrm{~g})$ with warm water taken for digestive and stomach problems; leaves and seeds are used as spices and condiment. 
Table 2 Bio-processing of medicinal plants of District Bageshwar (Continued)

\begin{tabular}{|c|c|}
\hline Scientific name & Mode of administration \\
\hline Trifolium repens $\mathrm{L}$. & Leaf paste $(5 \mathrm{~g})$ with water. \\
\hline Trigonella foemun-graecum L. & $\begin{array}{l}\text { Leaf juice is taken orally for curing obesity, indigestion, joints pain, and constipation; } 25 \mathrm{~g} \text { seeds are soaked } \\
\text { overnight filter; the filtrate taken orally in empty stomach for gastric problems and diabetes. }\end{array}$ \\
\hline Urtica dioica $\mathrm{L}$. & $\begin{array}{l}\text { Branches with leaves are gently rubbed on joints and muscles; fresh leaf twigs taken as vegetable; fine powder } \\
\text { of dry leaf }(5-10 \mathrm{~g}) \text { dissolve in } 50 \mathrm{ml} \text { water is taken orally in joints and muscular pain. }\end{array}$ \\
\hline Verbascum thapsus L. & Fresh leaf paste applied on affected part for boils; 8-10 whole plants mixed with grass given mulching animals. \\
\hline $\begin{array}{l}\text { Viola betonicifolia J.E. Smith } \\
\text { (Violaceae) }\end{array}$ & Paste of whole plant (fresh or semidry) applied on affected part for 1-2 weeks. \\
\hline Viola canescens Wall. Ex Roxb & Fresh plants (30-50) given with grass for 1 to 2 weeks. \\
\hline Vigna mungo L. & Paste prepared by grinding of $150 \mathrm{~g}$ seeds with water applied on the fractured part. \\
\hline Zanthoxylum armatum DC & Seeds $(100 \mathrm{~g})$ boiled with water taken orally twice a day; seed bark used as a spices. \\
\hline Zingiber officinale Rosc. & A piece $(5-10 \mathrm{~g})$ of broiled rhizome mixed with small amount of honey and chewed. \\
\hline
\end{tabular}

FYM farm yard manure, TS tablespoon

A correlation analysis was done among RFC, CI, UR, number of species used in treating different ailments, informant consensus factor (Fic), and medical importance. No evidence of any correlation was observed in most of the parameters; a highly positive correlation was only observed in the number of taxa used and the number of use reports (0.963). Also, there has been a moderately positive correlation observed between Fic and RFC which is of no significance in the study as both the parameters have been described differently.

Some species are also used in ethnoveterinary purposes for curing domestic animals. Ajuga parviflora is used to cure throat infection, Coriandrum sativum against poison, and Taraxacum officinale, Verbascum thapsus, and Viola canescens to increase lactation in milking animals.
The weakening of traditional ethnobotanical knowledge It is alarming to note that there has been a continued decline in traditional ethnobotanical knowledge in the target area (Fig. 7). An analysis of community perception on change in use pattern of medicinal plants in 2018 and a decade earlier (i.e., 2008) revealed that there is less number of species used for curing different ailments in recent years (Table 5). People are moving away from traditional herbal cure system, and the young generation has no interest in the traditional customs and values. Earlier, the people of remote areas preferred to consult with Vaidyas for primary healthcare, but in the last decade, since there is an increase in accessibility, availability, and affordability towards the allopathic medicinal system, the local community is also opting for such options. Despite that $57 \%$ of the total respondents believe that these plants are highly effective, 30\% found moderately effective, while only $13 \%$ feel it less effective

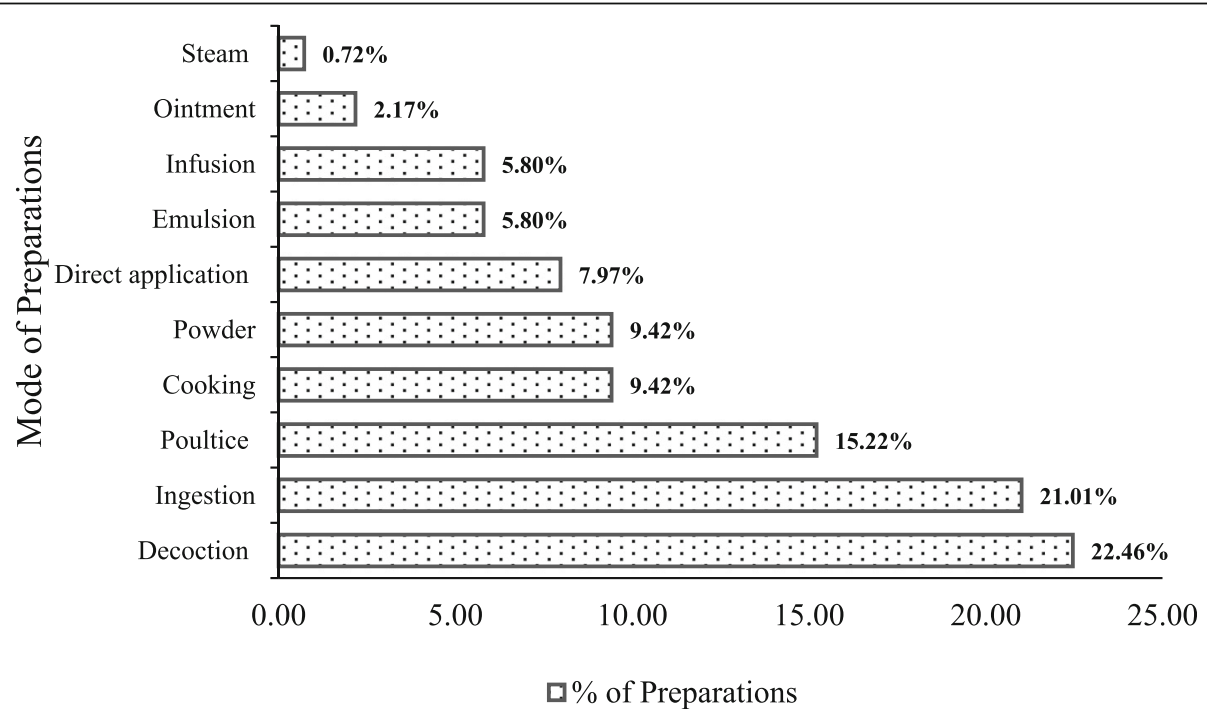

Fig. 5 Processing of plant parts in preparation of medicine 


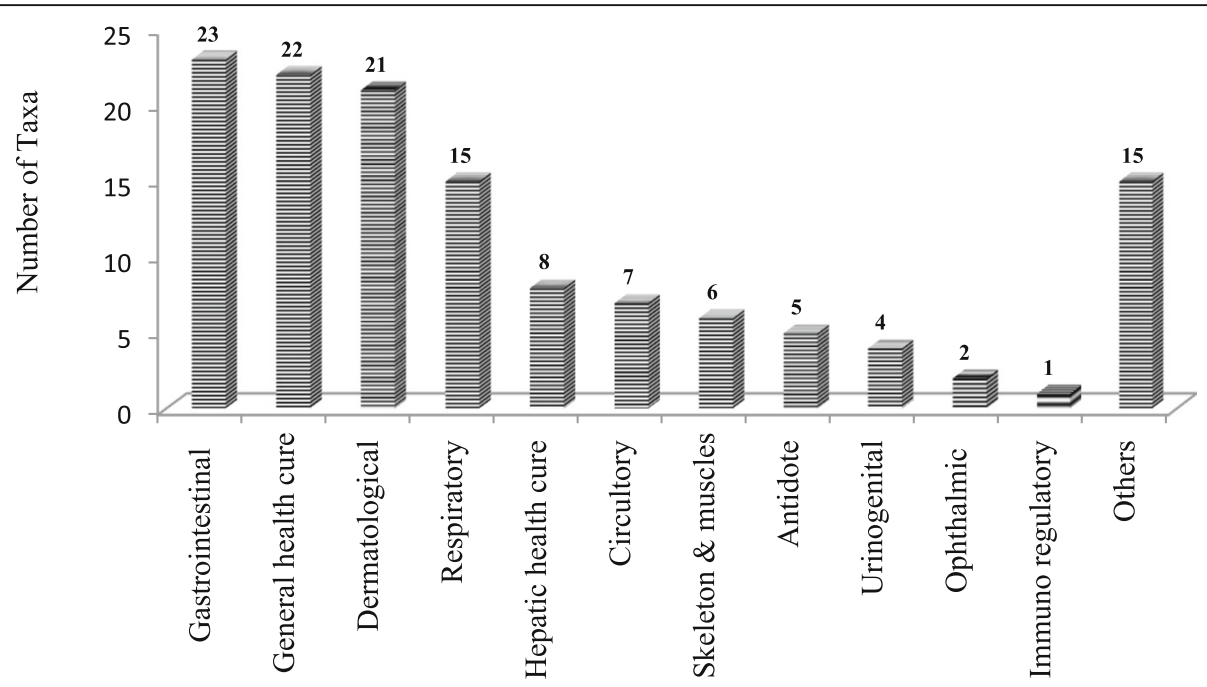

Fig. 6 Distribution of medicinal plants in different ailments category

Table 3 Use value of important ethnomedicinal species of target area

\begin{tabular}{|c|c|c|c|c|c|}
\hline Taxa & $U R^{a}$ & $\mathrm{FC}^{\mathbf{b}}$ & $\mathrm{Cl}^{\mathrm{c}}$ & NDAS & Ailments categories (decreasing order) \\
\hline Ocimum basilicum $\mathrm{L}$. & 97 & 88 & 0.97 & 5 & Respiratory, general health care, and others \\
\hline Cannabis sativa $\mathrm{L}$. & 94 & 63 & 0.94 & 6 & Gastrointestinal, others, and antidote \\
\hline Citrus aurantifolia (Christm) Sw. & 94 & 38 & 0.94 & 6 & Others, gastrointestinal, general health care, and respiratory \\
\hline Curcuma longa $\mathrm{L}$. & 91 & 78 & 0.91 & 5 & General health care, dermatological, and respiratory \\
\hline Setaria italica L. & 91 & 91 & 0.91 & 2 & Dermatological \\
\hline Angelica glauca Edgew. & 89 & 44 & 0.89 & 4 & Others and gastrointestinal \\
\hline Zingiber officinale Rosc. & 89 & 89 & 0.89 & 2 & Respiratory \\
\hline Ajuga parviflora Benth. & 87 & 56 & 0.87 & 5 & General health care, gastrointestinal, and urinogenital disorder \\
\hline Rephanus sativus L. & 87 & 87 & 0.87 & 1 & Hepatic health cure \\
\hline Emblica officinalis Gaertn. & 85 & 35 & 0.85 & 6 & Gastrointestinal, others, circulatory, and respiratory \\
\hline Glycine max (L.) Merri & 84 & 84 & 0.84 & 1 & Hepatic health cure \\
\hline Plantago ovate Forsk. & 83 & 74 & 0.83 & 3 & Gastrointestinal \\
\hline Ageratina adenophora (Spreng.) King \& H. Rob. & 80 & 80 & 0.80 & 2 & Dermatological \\
\hline Leucas lanata Benth & 80 & 80 & 0.80 & 1 & Respiratory \\
\hline Picrorhiza kurrooa Royle. & 80 & 53 & 0.80 & 2 & General health care and gastrointestinal \\
\hline Artemisia martima L. & 77 & 55 & 0.77 & 3 & Dermatological \\
\hline Zanthoxylum armatum DC & 77 & 61 & 0.77 & 5 & Others, general health care, respiratory, and gastrointestinal \\
\hline Acorus calamus L. & 74 & 55 & 0.74 & 3 & Others and skeleton and muscles \\
\hline Ajuga bracteosa Wall. ex Bent. & 72 & 55 & 0.72 & 3 & General health care, gastrointestinal, and urinogenital disorder \\
\hline Origanum vulgare L. & 71 & 31 & 0.71 & 5 & Dermatological, respiratory, general health care, and others \\
\hline Punica granatum L. & 71 & 59 & 0.71 & 4 & Respiratory, others, and hepatic health cure \\
\hline
\end{tabular}

NDAS no. of different ailment subcategories

${ }^{a}$ Total no. of use-reports of the taxon

bUse citation of taxa (the no. of informants that referred the taxon)

${ }^{\mathrm{c}} \mathrm{Cl}=\mathrm{UR} / N_{\mathrm{t}}$, where $N_{\mathrm{t}}$ is the total no. of reported taxa 
Table 4 Informant consensus factor $\left(F_{\mathrm{ic}}\right)$ and medicinal importance (MI) of ethnomedicinal plants

\begin{tabular}{|c|c|c|c|c|c|}
\hline Ailments category & No. of taxa $\left(\boldsymbol{N}_{\mathbf{t}}\right)^{\mathbf{a}}$ & Frequency $(\%)^{\mathbf{b}}$ & No. of use reports $\left(\boldsymbol{N}_{\mathbf{u r}}\right)$ & Informant consensus factor $\left(F_{i c}\right)^{c}$ & Medicinal importance $(\mathrm{MI})^{\mathbf{d}}$ \\
\hline Gastrointestinal & 23 & 32.86 & 695 & 0.97 & 30.22 \\
\hline General health cure & 22 & 31.43 & 524 & 0.96 & 23.82 \\
\hline Dermatological & 21 & 30.00 & 617 & 0.97 & 29.38 \\
\hline Respiratory & 15 & 21.43 & 402 & 0.97 & 26.80 \\
\hline Hepatic health cure & 8 & 11.43 & 364 & 0.98 & 45.50 \\
\hline Circulatory & 7 & 10.00 & 126 & 0.95 & 18.00 \\
\hline Skeleton and muscles & 6 & 8.57 & 178 & 0.97 & 29.67 \\
\hline Antidote & 5 & 7.14 & 83 & 0.95 & 16.60 \\
\hline Urinogenital & 4 & 5.71 & 137 & 0.98 & 34.25 \\
\hline Ophthalmic & 2 & 2.86 & 14 & 0.92 & 7.00 \\
\hline Immuno-regulatory & 1 & 1.43 & 15 & 1.00 & 15.00 \\
\hline Other & 15 & 21.43 & 377 & 0.96 & 25.13 \\
\hline
\end{tabular}

${ }^{a}$ No. of species listed in several of the categories of medicinal usage

${ }^{\mathrm{b}}$ Percentage of records on the total of 70 records

${ }^{c} F_{\text {ic }}=\left(N_{\text {ur }}-N_{\mathrm{t}}\right) /\left(N_{\mathrm{ur}}-1\right)$

${ }^{\mathrm{d}} \mathrm{Ml}=N_{\mathrm{ur}} / N_{\mathrm{t}}$

(Fig. 8). Interestingly, to cure selective diseases in children, such as Juga (removal of Ascaris), Chupad (heavy cough), and Kasar (constipation), still people prefer traditional cure systems as it has no side effects. During the study, it was observed that the Vaidyas do not share their knowledge; they believed that the treatment will not be effective if they share the knowledge with anybody. In the changing lifestyle and socioeconomic scenarios, most of the inhabitants are reluctant to live with their traditional heritage leading to the vanishing of the knowledge [58].

\section{Conclusions}

Community knowledge on the use and management of wild plant resources has always been integral to the survival, sustenance, and adaptation of human cultures $[47,53,58]$. This study revealed 70 medicinal plant species being used by the local marginal community of which 21 are the most extensively used species to treat various ailments. The significance of the traditional herbal healing system is highly relevant due to its effectiveness. It is cost-effective and based on local resources and still only means of cure for marginal communities in remote localities of Uttarakhand. With population growth and lack of health care, there is a need to adhere to the locally available resources to be utilized for general health care and provisioning of suitable side-effect free treatment to the communities. The community still uses these species; however, the level of use is decreasing because of upcoming modern allopathic based health care services. At the same time, there is also a decline in the number of local Vaidyas and herbal practitioners. This is because of increased access to modern hospitals and medicinal facilities in recent times.

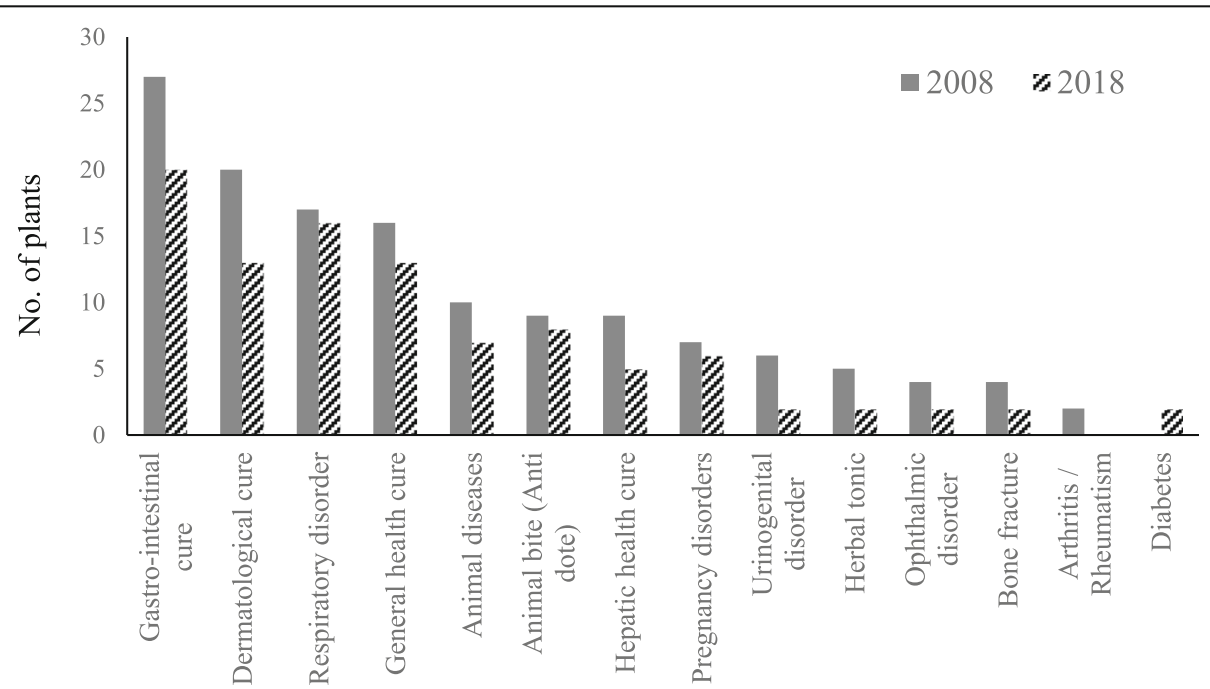

Fig. 7 Past (2008) and present (2018) use of plants in traditional health care system 
Table 5 Similarity between present and past ethnomedicinal uses of important species

\begin{tabular}{|c|c|c|}
\hline Botanical name & Use reports in study area & Earlier use reports from Uttarakhand \\
\hline $\begin{array}{l}\text { Aconitum heterophyllum } \\
\text { Wall. }\end{array}$ & Fever and vomiting & Fever, vomiting, and cough $[21,28,51,58]$ \\
\hline \multirow[t]{2}{*}{ Acorus calamus L. } & *Inflammation and insect repellent & Arthritis, cancer, convulsions, diarrhea \\
\hline & Sprain & Dyspepsia, epilepsy [41, 43]; sprain [50] \\
\hline $\begin{array}{l}\text { Ageratina adenophora } \\
\text { (Spreng.) }\end{array}$ & Cuts and wounds & Cuts and wounds $[31,41]$ \\
\hline \multirow{2}{*}{$\begin{array}{l}\text { Ajuga bracteosa Wall. ex } \\
\text { Benth. }\end{array}$} & ${ }^{*}$ Constipation & Fevers, diuretic [41] \\
\hline & Diuretic, fever & \\
\hline \multirow[t]{2}{*}{ Ajuga parviflora Benth. } & $\begin{array}{l}\text { *Constipation, stone, throat infection in animal } \\
\text { (Galghotu) }\end{array}$ & Headache, fever, stomach ache [51] \\
\hline & Fever, stomach ache & \\
\hline Allium sativum $\mathrm{L}$. & *Joint pain (arthritis) & Muscular pain $[43,52]$; ear pain [58] \\
\hline \multirow[t]{2}{*}{ Angelica glauca Edgew. } & *Spices and condiment and herbal tea & Constipation, bronchitis, and stomach \\
\hline & Stomach ache, vomiting & Disorders, vomiting $[31,43,50]$ \\
\hline Artemisia martima L. & Cuts, skin ailments, wounds & Skin ailments [51] \\
\hline $\begin{array}{l}\text { Asparagus racemosus } \\
\text { Willd. }\end{array}$ & *Stimulant, tonic, and stomach ache & Leucorrhoea, headache, hysteria, ulcer, liver disorders $[41,43]$ \\
\hline \multirow{2}{*}{$\begin{array}{l}\text { Berberis asiatica Roxb. ex } \\
\text { DC }\end{array}$} & *Fever & Diabetes, jaundice [41] \\
\hline & Diabetes & \\
\hline $\begin{array}{l}\text { Bergenia ciliata (Haw) } \\
\text { Sternb }\end{array}$ & Urinary infection and stone & $\begin{array}{l}\text { Fever, digestive disorders, skin diseases, urinary infection, and stone } \\
{[16,31]}\end{array}$ \\
\hline \multirow[t]{2}{*}{ Cannabis sativa $\mathrm{L}$. } & $\begin{array}{l}\text { *Insect bite, stomach ache, purgative and laxative, } \\
\text { warm effect in winters }\end{array}$ & Analgesic, cough, cold, sedative, narcotic, skin diseases [43] \\
\hline & Carminative, constipation & \\
\hline Centella asiatica $\mathrm{L}$. & *Headache & Inflammatory infections, wounds $[41,43]$ \\
\hline $\begin{array}{l}\text { Citrus aurantifolia } \\
\text { (Christm) Sw. }\end{array}$ & $\begin{array}{l}\text { Cold, constipation, headache, herbal tea, source of } \\
\text { vitamin " } \mathrm{C} \text {," and weight loss }\end{array}$ & Diarrhea, dysentery, fever, headache [53] \\
\hline Citrus hystrix DC. & $\begin{array}{l}\text { *Against poison, cold, removal of Ascaris (anti- } \\
\text { parasitic) }\end{array}$ & Vomiting [52] \\
\hline Coriandrum sativum L. & ${ }^{*}$ Against poison & Stomachic and diuretic [43] \\
\hline \multirow[t]{2}{*}{ Curcuma longa $\mathrm{L}$. } & *Internal injury & Skin disorders, wound healing $[43,52]$ \\
\hline & Cough, cuts and wounds, and cosmetics & \\
\hline $\begin{array}{l}\text { Cynoglossum zeylanicum } \\
\text { Thunb. ex Lehm. }\end{array}$ & *Boils & Asthma, bronchitis, cough, vomiting $[16,54]$ \\
\hline $\begin{array}{l}\text { Dactylorhiza hatagirea } \\
\text { (D.Don)Soo }\end{array}$ & Bleeding and wounds & Burns, cuts, checks bleeding $[31,41]$ \\
\hline Dioscorea deltoidea Wall & Cough and cold & Cough, fever, urinogenital disorders $[31,41,43,51]$ \\
\hline $\begin{array}{l}\text { Drymaria cordata (L.) } \\
\text { Willd. ex Schult }\end{array}$ & ${ }^{*}$ Cough & Laxative [49]; bile complaints [51] \\
\hline $\begin{array}{l}\text { Duchesnea indica } \\
\text { (Andrews) Focke }\end{array}$ & *Burns and removal of burn scars & Diarrhea, fever, leucorrhoea [54]; skin diseases [53] \\
\hline $\begin{array}{l}\text { Emblica officinalis } \\
\text { Gaertn. }\end{array}$ & $\begin{array}{l}\text { Diabetes, purgative and laxative, carminative, } \\
\text { stomach ache, and source of vitamin " } \mathrm{C} \text { " }\end{array}$ & $\begin{array}{l}\text { Asthma, digestive disorders, hair fall [31]; dysentery, cholera, and } \\
\text { jaundice }[41,51]\end{array}$ \\
\hline $\begin{array}{l}\text { Euphorbia prolifera } \\
\text { Ehrenb. ex Boiss }\end{array}$ & *Insect repellent & -- \\
\hline Ficus palmata Forsk. & ${ }^{*}$ Cuts and wounds & Lungs diseases, skin diseases $[43,49,51]$ \\
\hline Ficus roxburghii Wall. & *Acidity, source of vitamin "C" & Laxative [49] \\
\hline Glycine max (L.) Merri & *Jaundice & -- \\
\hline
\end{tabular}


Table 5 Similarity between present and past ethnomedicinal uses of important species (Continued)

\begin{tabular}{|c|c|c|}
\hline Botanical name & Use reports in study area & Earlier use reports from Uttarakhand \\
\hline $\begin{array}{l}\text { Hedychium spicatum } \\
\text { Buch. Ham. ex Smith. }\end{array}$ & $\begin{array}{l}\text { Anti-lice, cough, cosmetics, intestinal problems, } \\
\text { purgative and laxative, carminative }\end{array}$ & $\begin{array}{l}\text { Carminative, stomachic, liver complaints, fevers, vomiting, diarrhea, } \\
\text { inflammation, snake bite }[16,41,51]\end{array}$ \\
\hline Hordium vulgare $\mathrm{L}$. & *Burns, warm, and nutritive effect & -- \\
\hline Leucas lanata Benth & ${ }^{*}$ Cough & Cuts, to check bleeding, wounds [51] \\
\hline Mentha arvensis L. & Stomach ache and vomiting & Diarrhea, stomach ache $[51,55]$ \\
\hline Micromeria biflora Benth. & *Fever & Joints pain, worm infested wounds [41] \\
\hline $\begin{array}{l}\text { Microtyloma uniflorum } \\
\text { (Lam) Verdc. }\end{array}$ & Stone & Stone [52] \\
\hline Momordica charantia L. & Diabetes & Jaundice, diabetes [43] \\
\hline Ocimum basilicum L. & $\begin{array}{l}\text { Cough and cold, fever, herbal tea, warm effect in } \\
\text { winters }\end{array}$ & Cough, cold, fever [16] \\
\hline Origanum vulgare $\mathrm{L}$. & Cough and cold, fever, herbal tea, and wounds & Cold, diarrhea, fever, indigestion, influenza, menstrual disorder $[43,51]$ \\
\hline Picrorhiza kurrooa Royle. & Abdominal pain, fever & $\begin{array}{l}\text { Anemia, asthma, blood troubles, inflammation, jaundice [41]; fever, } \\
\text { stomach ache [31]; abdominal pain, cataract }[50,51]\end{array}$ \\
\hline Plantago ovate Forsk. & Constipation, digestive problems, and diarrhea & Constipation, dysentery, and diarrhea [41] \\
\hline Plantego lanceolata $\mathrm{L}$. & ${ }^{*}$ Removal of stomach worm of domestic animals & $\begin{array}{l}\text { Dyspepsia, sore wounds, dysentery, purgative, mouth disease, and } \\
\text { chicks [41] }\end{array}$ \\
\hline $\begin{array}{l}\text { Podophyllum hexandrum } \\
\text { Royle }\end{array}$ & Wounds & Purgative, cancer [41]; wounds [31] \\
\hline $\begin{array}{l}\text { Polygonatum cirrhifolium } \\
\text { (Wall.) }\end{array}$ & *Blood purifier, cuts, tonic, and wounds & Anemia, fever, bronchitis, general debility [ 54] \\
\hline $\begin{array}{l}\text { Polygonatum } \\
\text { verticillatum } \mathrm{L} .\end{array}$ & Carminative and wounds & Aphrodisiac, gastric complaints, nervine tonic, wound healing $[43,51]$ \\
\hline Prunus persica Stokes. & *Headache & Ear infection of children [31]; antipyretic, brain tonic [21] \\
\hline Psidium guajava $\mathrm{L}$. & Mouth blisters (astringent) & Mouth blisters $[51,59]$ \\
\hline Punica granatum L. & *Anemia, cough, cold, source of vitamin "C" & Diarrhea, dysentery, piles [41] \\
\hline Ranunculus repens $\mathrm{L}$. & *Boils and intestinal pains (Nas Palatana) & -- \\
\hline Rephanus sativus L. & Jaundice & Jaundice [52] \\
\hline Rheum emodi Wall. & Fever and wounds & Cuts, fracture, wounds [56] \\
\hline $\begin{array}{l}\text { Rhododendron arboreum } \\
\text { Smth }\end{array}$ & Liver complaints, tonic & Heart tonic [31], stomach diseases [41] \\
\hline Rosa moschata Hermm. & *Boils, cuts, eye diseases, wounds & Leucorrhoea, bleeding, pregnancy termination [16] \\
\hline Rubia cordifolia L. & *Fever & Blood purifier, joints pain, leucorrhoea, cuts, wounds, insect sting [51] \\
\hline Rubus ellipticus Smith. & *Fever and stomach ache & Blood pressure, diarrhea [41] \\
\hline $\begin{array}{l}\text { Saussurea costus (Falc.) } \\
\text { Lipsch. }\end{array}$ & Cough, dysentery, fever, stomach ache & Asthma, cough, dysentery, fever $[51,55]$; abdominal pain [58] \\
\hline Setaria italic L. & ${ }^{*}$ Chicken pox and measles & -- \\
\hline $\begin{array}{l}\text { Silene vulgaris (Moench) } \\
\text { Garcke }\end{array}$ & ${ }^{*}$ Fever and removal of Ascaris (anti-parasitic) & Asthma, bronchitis [16] \\
\hline \multirow{2}{*}{$\begin{array}{l}\text { Swertia angustifolia } \\
\text { Buch.-Ham. ex D.Don. }\end{array}$} & *Skin ailments & Pneumonia, cold, cough, fever [51] \\
\hline & Fever & \\
\hline $\begin{array}{l}\text { Taraxacum officinale } \\
\text { Weber. }\end{array}$ & $\begin{array}{l}\text { *Snake bite and to increase lactation in mulching } \\
\text { animals }\end{array}$ & Headache, acts as a heart tonic and blood purifier $[28,58]$ \\
\hline Tegetus erecta $\mathrm{L}$. & *Ear infection, fever, and wounds & Muscular pain, piles, ulcer, wound healing [43] \\
\hline $\begin{array}{l}\text { Terminalia chebula } \\
\text { (Gaertner) Retz. }\end{array}$ & $\begin{array}{l}\text { Carminative, constipation, digestive problems, } \\
\text { diarrhea, purgative }\end{array}$ & Asthma, digestive problems, diarrhea, purgative $[16,31]$ \\
\hline Thalictrum foliosum DC. & *Eye infection (white-dot-cataract), insect repellent & Gastric trouble, used to control external parasites [41] \\
\hline
\end{tabular}


Table 5 Similarity between present and past ethnomedicinal uses of important species (Continued)

\begin{tabular}{|c|c|c|}
\hline Botanical name & Use reports in study area & Earlier use reports from Uttarakhand \\
\hline Botanical name & Uses report in study area & Earlier uses report from Uttarakhand \\
\hline \multirow[t]{2}{*}{ Thymus serpyllum L. } & ${ }^{*}$ Asthma, joint pain, spices, and condiments & Laxative, stomachic [41]; cough, epilepsy, itching, and skin diseases \\
\hline & Digestive and stomach problems & Menstrual disorders, swelling [51] \\
\hline Trifolium repens $\mathrm{L}$. & *Headache and skin disease of dogs & Astringent [16] \\
\hline $\begin{array}{l}\text { Trigonella foemun- } \\
\text { graecum } L \text {. }\end{array}$ & $\begin{array}{l}\text { Carminative, constipation, diabetes, indigestion, } \\
\text { joint pain, and obesity }\end{array}$ & Diabetes, rheumatism $[16,52]$ \\
\hline Urtica dioica $\mathrm{L}$. & *Joint pain, warm and nutritive effect & Skin diseases, boils [31, 41]; bone fracture [51] \\
\hline \multirow[t]{2}{*}{ Verbascum thapsus L. } & *To increase lactation in milching animals & Cough, fever, rheumatism [41]; boils eye cataract [51] \\
\hline & Boils & \\
\hline $\begin{array}{l}\text { Viola betonicifolia J.E. } \\
\text { Smith }\end{array}$ & *Snake bite & Blood diseases, cough, fever, skin [57] \\
\hline $\begin{array}{l}\text { Viola canescens Wall. Ex } \\
\text { Roxb }\end{array}$ & *To increase lactation in milching animals & Cough, cold, malaria, jaundice $[43,49]$ \\
\hline Vigna mungo L. & *Fracture & -- \\
\hline $\begin{array}{l}\text { Zanthoxylum armatum } \\
\text { DC }\end{array}$ & $\begin{array}{l}\text { Carminative, cough and cold, toothache, spices } \\
\text { and condiments }\end{array}$ & Toothache [31]; constipation, gastric disorders $[41,43,50]$ \\
\hline $\begin{array}{l}\text { Zingiber officinale Rosc. } \\
\text { (Zingiberaceae) }\end{array}$ & Cough and cold & Asthma, cough, and cold [43] \\
\hline
\end{tabular}

${ }^{*}$ New ethnomedicinal use reports documented from study sites

This possesses a significant challenge to the continuity of the traditional herbal cure system. The impoverishment of such knowledge may lead to an enormous loss to the scientific community. The ethnomedicinal knowledge and information provided in this study are of significant value for scientific validation, product development, conservation, and policy planners for sustainable management of medicinal plants and traditional herbal cure system. It is suggested to explore and establish linkage between traditional health practices and modern health care systems. It can be done by testing bioactive compound and biological activity of most preferred plant species and assessing the safety and efficacy of the local herbal formulation. Such an investigation may lead to many new and novel drug discovery. It is also recommended that

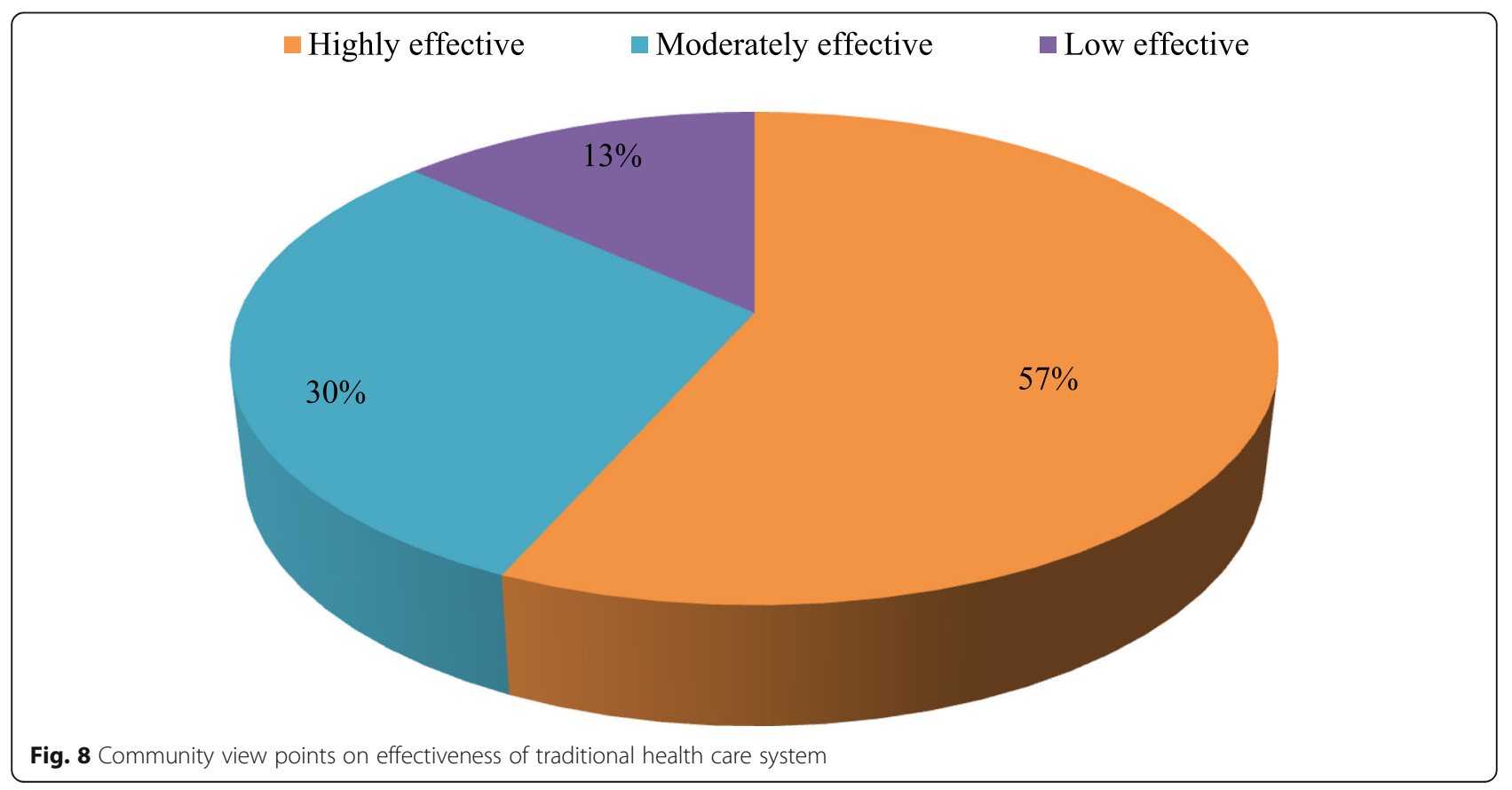


the natural habitats of medicinal plants should be protected for the conservation of valuable gene pool and to control the exploitation of species. Since ethnomedicinal information is strongly linked to local livelihoods, culture, and environment, it is strongly recommended to further continue studying the subject to serve humanity with healthier and operative health care measures.

\section{Acknowledgements}

We owe our gratitude to the people of Garur-Ganga valley and Saryu valley of District Bageshwar, Uttarakhand, who shared the valuable information and knowledge. The authors thankfully acknowledge the facilities received from GBPNIHE, Kosi-Katarmal, Almora, India, for undertaking this work. We are thankful to DST, Govt. of India for the financial assistance provided under a NMSHE, Task Force 5 sponsored project entitled "Network program on the convergence of traditional knowledge system for sustainable development in the Indian Himalayan Region." We sincerely thank Dr Deepshikha Arya, Research Officer, CCRAS-RARI, Ranikhet, for her support to help in identifying the plant species, as well as Prof. S.C. Garkoti, JNU for his constant support and cooperation.

\section{Authors' contributions}

SNO, DT, and AA planned and performed the study and field survey, wrote the draft manuscript, and analyzed the data, and RCS revised the manuscript and data analysis. All authors read and approved the final manuscript.

\section{Funding}

The study has been funded by Department of Science and Technology, Govt. of India under National Action Plan for Climate Change (NAPCC) through National Mission on Sustaining Himalayan Ecosystems (Task Force 5Network programme on the convergence of traditional knowledge system for sustainable development in the Indian Himalayan Region).

\section{Availability of data and materials}

The authors already included all data in the manuscript collected during the field surveys. The documented medicinal plant species were deposited at Centre of Socio-economic Development (CSED), GBPNIHE, Kosi-Katarmal, Almora, Uttarakhand.

\section{Ethics approval and consent to participate}

During field work, prior consent of the informants was taken conducting these studies. This was done to adhere to the ethical standards of community participation in scientific research.

\section{Consent for publication}

Not applicable.

\section{Competing interests}

The authors declare that they have no competing interests.

Received: 13 December 2019 Accepted: 12 May 2020

Published online: 24 May 2020

\section{References}

1. Kala CP. Medicinal plants conservation and enterprise development. Med Plants. 2009;1(2):79-95.

2. L.K Rai LK, Prasad P, Sharma E. Conservation threats to some important medicinal plants of Sikkim Himalaya. Biol Conser. 2000; 93(1):27-33.

3. Kala CP, Dhyani PP, Sajwan BS. Developing the medicinal plants sector in northern India: challenges and opportunities. J Ethnobiol Ethnomed. 2006:2(1):32.

4. Caniago I, Siebert S. Medicinal plants ecology, knowledge and conservation in Kalimantan, Indonesia. Econ Bot. 1998; 52:229-250.

5. Kuniyal CP, Bisht VK, Negi JS, Bhatt VP, Bisht DS, Butola JS, Sundriyal RC, Singh SK. Progress and prospect in the integrated development of medicinal and aromatic plants (MAPs) sector in Uttarakhand. Western Himalaya. Environ Develop Sustaina. 2015;17(5):1141-62.

6. Rossato SC, Leitao-Filho H, Gegossi A. Ethnobotany of caicaras of the Atlantic forest coast (Brazil). Econ Bot. 1999;53:387-95.
7. Hanazaki N, Tamashiro JY, Leitao-Filho H, Gegossi A. Diversity of plant uses in two caicaras communities from the Atlantic forest coast. Brazil. Biodivers Conserv. 2000;9:597-615.

8. Gazzaneo LR, Paiva de Lucena RF, Paulino de Albuquerque U. Knowledge and use of medicinal plants by local specialists in a region of Atlantic Forest in the state of Pernambuco (Northeastern Brazil). J Ethnobiol Ethnomed. 2005; 1(1):9

9. Saha D, Sundriyal M, Sundriyal RC. Diversity of food composition and nutritive analysis of edible wild plants in multi-ethnic tribal land, Northeast India: an important facet for food supply. Indian Journal of Traditional Knowledge. 2014;13(4):698-705.

10. Kala CP. Status and conservation of rare and endangered medicinal plants in the Indian trans-Himalaya. Biol Conserv. 2000;93:371-9.

11. Bisht VK, Kandari LS, Negi JS, Bhandari AK, Sundriyal RC. Traditional use of medicinal plants in district Chamoli, Uttarakhand. India. Jour Med PI Res. 2013;7(15):918-29.

12. Kala CP. Current status of medicinal plants used by traditional Vaidyas in Uttaranchal State of India. Ethnobotany Research Applications. 2005;3:267-78.

13. Osmaston AE. A forest flora for Kumaun. Dehradun, India: International Book Distributors; 1926

14. Naithani BD. Flora of Chamoli. Botanical Survey of India, Vol. 1 and 2. Dehradun, India. 1985

15. Kirtikar KR, Basu BD. Indian medicinal plants. Bishan Singh Mahendra Pal Singh, Dehradun. 1994;1994.

16. Gaur RD. Flora of the District Garhwal: North West Himalaya (with ethnobotanical notes). Srinagar, Garhwal: Transmedia; 1999.

17. Phillips O, Gentry AH, Reynel C, Wilkin P, Galvez DBC. Quantitative ethnomedicine and Amazonian conservation. Biodivers Conserv Biol. 1994;8:225-48.

18. Tardio J, Pardo-de-Santayana M. Cultural importance indices: a comparative analysis based on the useful wild plants of southern Cantabria (northern Spain). Econ Bot. 2008;62(1):24-39.

19. Pardo-de-Santayana M, Tardio J, Blanco E, Carvalho AM, Lastra JJ, San Miguel E, Morales R. Traditional knowledge of wild edible plants used in the northwest of the Iberian Peninsula (Spain and Portugal): a comparative study. J Ethnobiol Ethnomed. 2007;3:27.

20. Trotter RT, Logan MH. Informant consensus: a new approach for identifying potentially effective medicinal plants. In: Etkin NL, editor. Plants in indigenous medicine and diet: biobehavioural approaches. Bedfort hills, New York: Redgrave Publishers. 1986; 91-112.

21. Gurdal B, Kultur S. An ethnobotanical study of medicinal plants in Marmaris (Mugla, Turkey). J Ethnopharmacol. 2013;146(1):113-26.

22. Ahmad M, Sultana S, Fazl-i-Hadi S, Ben Hadda T, Rashid S, Zafar M, Khan MA, Khan MPZ, Yaseen G. An ethnobotanical study of medicinal plants in high mountainous region of Chail valley (district swat-Pakistan). J Ethnobiol Ethnomed. 2014;10(1):36.

23. Tugume P, Kakudidi EK, Buyinza M, Namaalwa J, Kamatenesi M, Mucunguzi $P$, Kalema J. Ethnobotanical survey of medicinal plant species used by communities around Mabira Central Forest Reserve. Uganda. J EthnobiolEthnomed. 2016;12(1):5

24. Umair M, Altaf M, Abbasi AM. An ethnobotanical survey of indigenous medicinal plants in Hafizabad district. Punjab-Pakistan. PloS one. 2017;12(6):e0177912.

25. Farooq A, Amjad MS, Ahmad K, Altaf M, Umair M, Abbasi AM. Ethnomedicinal knowledge of the rural communities of Dhirkot, Azad Jammu and Kashmir. Pakistan. J Ethnobiol Ethnomed. 2019;15:45.

26. El Amri J, El Badaoui K, Zair T, Bouharb H, Chakir S, Alaoui T. Ethnobotanical study of medicinal plants in the region El Hajeb (Central Morocco). J Res Biol. 2015:4(8):1568-80.

27. Boudjelal A, Henchiri C, Sari M, Sarri D, Hendel N, Benkhaled A, Ruberto G. Herbalists and wild medicinal plants in M'Sila (North Algeria): an ethnopharmacology survey. J Ethnopharmacol. 2013;148(2):395-402.

28. Singh $\mathrm{H}$. Importance of local names of some useful plants in ethnobotanical study. Indian J Tradit Knowledge. 2008;7(2):365-70.

29. Shandesh B, Chaudhary RP, Quave CL, Taylor RSL. The use of medicinal plants in the transhimalayan arid zone of Mustang district. Nepal. J Ethnobiol Ethnomed. 2010;6:14

30. Kumar M, Mehraj A, Sheikh MA, Bussmann RW. Ethnomedicinal and ecological status of plants in Garhwal Himalaya. India. J Ethnobiol Ethnomed. 2011;7:32.

31. Malik ZA, Bhat JA, Ballabha A, Bussmann RW. Ethnomedicinal plants traditionally used in health care practices by inhabitants of Western Himalaya. J Ethnopharmacolog. 2015;172:133-44. 
32. Bhat JA, Kumar M, Bussmann RW. Ecological status and traditional knowledge of medicinal plants in Kedarnath Wildlife Sanctuary of Garhwal Himalaya. India. J. Ethnobiol Ethnomed. 2013;9:1.

33. Kunwar RM, Nepal BK, Kshetri HB, Rai SK, Bussmann RW. Ethnomedicine in Himalaya: a case study from Dolpa, Humla. Jumla and Mustang districts of Nepal. J. Ethnobiol Ethnomed. 2006;2:27.

34. Kunwar RM, Shrestha KP, Bussmann RW. Traditional herbal medicine in Farwest Nepal: a pharmacological appraisal. J. Ethnobiol Ethnomed. 2010;6:35.

35. Kunwar RM, Mahat L, Acharya RP, Bussmann RW. Medicinal plants, traditional medicine, markets and management in far-west Nepal. J. Ethnobiol. Ethnomed. 2013;9:24.

36. Moore PD. Trials in bad taste. Nature. 1994;370:410-1.

37. Keter LK, Mutiso PC. Ethnobotanical studies of medicinal plants used by traditional health practitioners in the management of diabetes in lower eastern province. Kenya. J. Ethnopharmacol. 2012;139:74-80.

38. Quave CL, Pieroni AA. Reservoir of ethnobotanical knowledge informs resilient foodsecurity and health strategies in the Balkans. Nature Plants. 2015;1(2):14021.

39. Mahmood A, Mahmood A, Malik RN, Shinwari ZK. Indigenous knowledge of medicinal plants from Gujranwala district. Pakistan. J Ethnopharmacol. 2013; 148(2):714-23.

40. Bano A, Ahmad M, Hadda TB, Saboor A, Sultana S, Zafar M, Khan MPZ, Arshad M, Ashraf MA. Quantitative ethnomedicinal study of plants used in the skardu valley at high altitude of Karakoram-Himalayan range. Pakistan. J Ethnobiol Ethnomed. 2014;10(1):43.

41. Bisht VK, Rana CS, Negi JS, Bhandari AK, Purohit V, Kuniyal CP, Sundriyal RC. Lamiaceous ethno-medico-botanicals in Uttarakhand Himalaya. India. Jour Med PI Res. 2012;6(26):4281-91.

42. Sundriyal RC. Medicinal plant cultivation and conservation in the Himalaya: an agenda for action. Indian Forester. 2005;131(3):410-24.

43. Singh P, Attri BL. Survey on traditional uses of medicinal plants of Bageshwar valley (Kumaun Himalaya) of Uttarakhand. India. Intern J Conserv Sci. 2014;5(2):223-34.

44. Tewari S, Paliwal AK, Joshi B. Medicinal use of some common plants among people of Garur Block of District Bageshwar, Uttarakhand. India. Octa J Biosci. 2014;2(1):32-5.

45. Bhatt D, Arya D, Chopra N, Upreti BM, Joshi GC, Tewari LM. Diversity of ethnomedicinal plant: a case study of Bageshwar district Uttarakhand. Journal of Medicinal Plants Studies. 2017;5(2):11-24.

46. Tuttolomondo T, Licata M, Leto C, Bonsangue G, Gargano ML, Venturella G, La Bella S. Popular uses of wild plant species for medicinal purposes in the Nebrodi Regional Park (north-eastern Sicily, Italy). J Ethnopharmacol. 2014; 157:21-37.

47. Jain A, Sundriyal M, Roshnibala S, Kotoky R, Kanjilal PB, Singh HB, Sundriyal RC. Dietary use and conservation concern of edible wetland plants at IndoBurma hotspot: a case study from northeast India. Jour Ethnobiol Ethnomed. 2011;7:7-29.

48. Pandey K, Pandey S. Indigenous medicines of Raji tribes of Uttarakhand. Indian J Tradit Knowledge. 2010;9(1):131-3.

49. Joshi M, Kumar M, Bussmann RW. Ethnomedicinal uses of plant resources of the Haigad watershed in Kumaun Himalaya. J Medicinal and Aromatic Plant Science and Biotechnology. 2010;4(1):43-6.

50. Samal PK, Dhyani PP, Dollo M. Indigenous medicinal practices of Bhotia tribal community in Indian Central Himalaya. Indian J Tradit Knowledge. 2010:9(1):140-4.

51. Saha D, Sundriyal RC. Utilization of non-timber forest products in humid tropics: consumption pattern, contribution to rural income and forest revenue. Forest Policy and Economics. 2012;14:28-40.

52. Samal PK, Shah A, Tiwari SC, Agrwal DK. Indigenous healthcare practices and their linkages with bioresources conservation and socio-economic development in Central Himalayan region of India. Indian J Tradit Knowledge. 2004;3(1):12-26.

53. Singh A, Nautiyal MC, Kunwar RM, Bussmann RW. Ethnomedicinal plants used by local inhabitants of Jakholi block, Rudraprayag district, Western Himalaya. India. J Ethnobiol Ethnomed. 2017;13:49.

54. Uprety Y, Poude RC, Gurung J, Chettri N, Chaudhary RP. Traditional use and management of NTFPs in Kangchenjunga Landscape: implications for conservation and livelihoods. Jour Ethnobiol Ethnomed. 2016:12:19.

55. Jalal JS, Garkoti SC. Medicinal plants used in the cure of stomach disorders in Kumaon Himalaya, Uttarakhand. India. Academia J Medicinal Plants. 2013; 1(7):116-21.
56. Garbyal SS, Aggarwal KK, Babu CR. Traditionally used medicinal plants in Dharchula Himalayas of Pithoragarh district. Uttaranchal. Indian J Tradit Knowledge. 2005;4(2):199-207.

57. Mehta PS, Negi KS, Ojha SN, Rayal A, Verma SK. Herbal based traditional practices used by the Bhotias and Gangwals of the central Himalayan region, Uttarakhand. India. J. Nanotechnol. 2013;2(1):83-96.

58. Maikhuri RK, Nautiyal S, Rao KS, Saxena KG. Medicinal plant cultivation and biosphere reserve management: a case study from the Nanda Devi Biosphere Reserve. Himalaya. Curr Sci. 1998;74(2):157-63.

\section{Publisher's Note}

Springer Nature remains neutral with regard to jurisdictional claims in published maps and institutional affiliations.
Ready to submit your research? Choose BMC and benefit from:

- fast, convenient online submission

- thorough peer review by experienced researchers in your field

- rapid publication on acceptance

- support for research data, including large and complex data types

- gold Open Access which fosters wider collaboration and increased citations

- maximum visibility for your research: over $100 \mathrm{M}$ website views per year

At $\mathrm{BMC}$, research is always in progress.

Learn more biomedcentral.com/submissions 